\title{
Unsymmetrical Heptamethine Dyes for NIR Dye-Sensitized Solar Cells
}

\author{
Thomas Geiger, ${ }^{1}$ Iuliia Schoger, ${ }^{1}$ Daniel Rentsch, ${ }^{1}$ Anna Christina Véron, ${ }^{1}$ \\ Frédéric Oswald, ${ }^{2}$ Toby Meyer, ${ }^{2}$ and Frank Nüesch ${ }^{1,3}$ \\ ${ }^{1}$ Empa, Swiss Federal Laboratories for Materials Science and Technology, Laboratory for Functional Polymers, \\ Überlandstrasse 129, 8600 Dübendorf, Switzerland \\ ${ }^{2}$ Solaronix, Rue de l'Ouriette 129, 1170 Aubonne VD, Switzerland \\ ${ }^{3}$ Institut des Matériaux, Ecole Polytechnique Fédéral de Lausanne, EPFL Station 12, 1015 Lausanne, Switzerland
}

Correspondence should be addressed to Thomas Geiger; thomas.geiger@empa.ch

Received 9 April 2014; Accepted 8 May 2014; Published 4 June 2014

Academic Editor: Vincenzo Augugliaro

Copyright (C) 2014 Thomas Geiger et al. This is an open access article distributed under the Creative Commons Attribution License, which permits unrestricted use, distribution, and reproduction in any medium, provided the original work is properly cited.

Seven unsymmetrical heptamethine dyes with carboxylic acid functionality were synthesized and characterized. These nearinfrared dyes exhibit outstanding photophysical properties depending on their heterocyclic moieties and molecular structure. As proof of principle, the dyes were used as photosensitizers in dye-sensitized solar cells. Using the most promising dye, an overall conversion efficiency of $1.22 \%$ and an almost colorless solar cell were achieved.

\section{Introduction}

In order to achieve chromophores which absorb light in the far red or even in the near-infrared region (NIR), increased efforts were made by developing new molecular dye structures and synthetic strategies or by investigating basic structure property relationships. Intense absorption in the NIR is mandatory for many technologies and applications, for example, energy conversion in photovoltaic cells [1]. However, while many dyes provide absorption from the visible to the NIR $[2,3]$ range, only few absorb in the NIR only and are able to convert energy efficiently. Particularly for application in dye-sensitized solar cells (DSCs), only a few dye classes with appropriate molecular design are known to be viable NIR absorbers: squaraine dyes, croconine dyes, diketopyrrolopyrrole derivatives, and polymethine dyes with a single absorption maximum, $\lambda_{\max }$, above $700 \mathrm{~nm}$. Recently, Park et al. comprehensively reviewed the NIR sensitization in DSCs [4]. Polymethine dyes [5], in particular, the subclass of heptamethine dyes, exhibit outstanding photophysical properties, for example, absorption maximum $\left(\lambda_{\max }\right)$ above $700 \mathrm{~nm}$ with high molar extinction coefficient $(\varepsilon)$ of over $100000 \mathrm{Lmol}^{-1} \mathrm{~cm}^{-1}$. Furthermore, heptamethine dyes can be obtained via straightforward and cost-effective synthesis in high yields in contrast to metal complex photosensitizers. In this context, several publications reported about synthesis and first applications of heptamethine dyes in DSCs [6-11]. In common with all reported heptamethine dyes, the essential carboxylic acid anchor group was symmetrically positioned either at the heterocyclic moieties or the alkyl chains which are substituted to the nitrogen atoms of the heterocycles or at the cyclohexene moiety of the polymethine chain.

Following the concept and molecular design of unsymmetrical squaraine dyes which were synthesized and successfully applied [12-15] in our previous works [16-18], we report on the synthesis, characterization, and application of unsymmetrical heptamethine dyes Heptal-Hepta7 with one carboxylic acid anchor group (Figure 1). Here, the anchor group is in conjugation with one of the heterocyclic moieties (indole- or quinoline-derivative). The polymethine core is rigidified by a cyclohexene moiety which is substituted by a chlorine atom or phenyl-group.

The second terminating heterocycle (benzindole-, quinoline-, and thiazole-derivative) is chemically different from the first heterocycle carrying the anchor group. In order to improve solubility in organic solvents and to hinder dye 


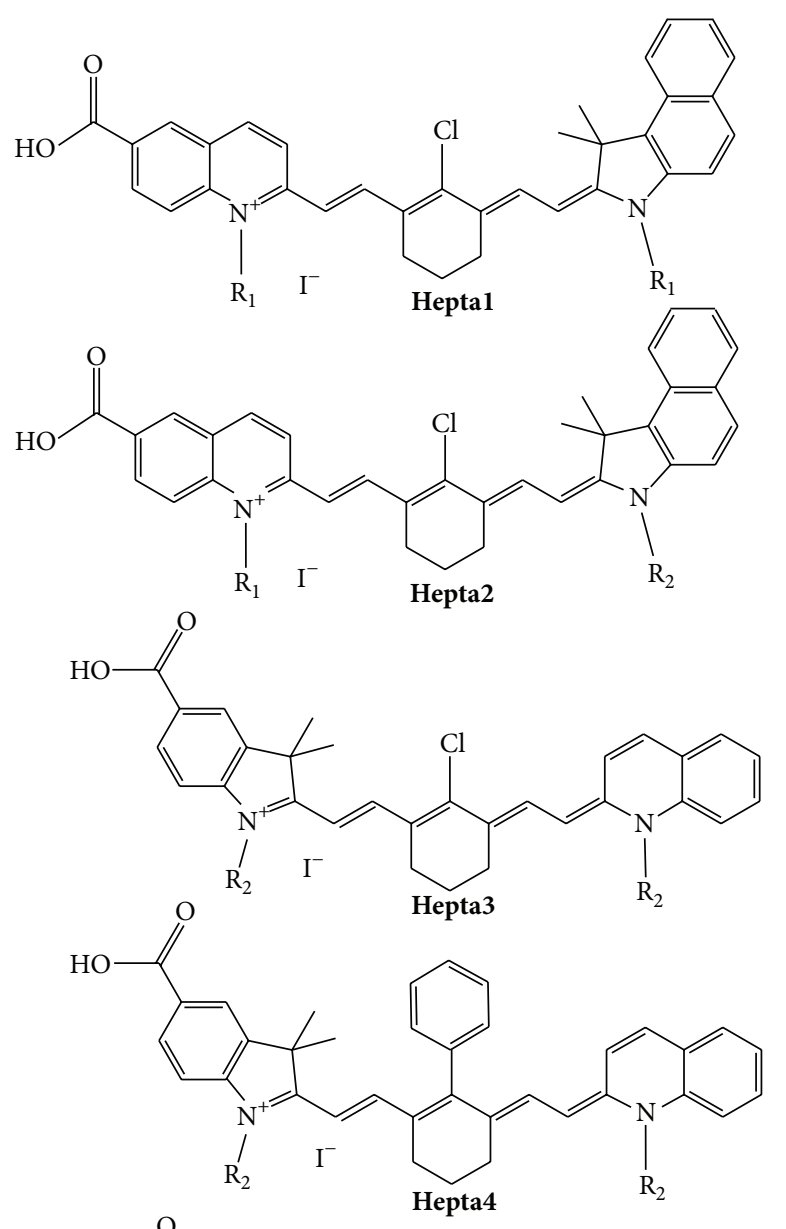<smiles>[R16]C1CCC(C=CC2=C(Cl)C(=CC=C3C=CN([R2])c4ccccc43)CC2)=[N+]([R1])c2ccc(C(=O)O)cc21</smiles><smiles>[R1]N1C(=CC=C2CCCC(=C[I-]C3=[N+]([R1])c4ccc(C(=O)O)cc4C3(C)C)C2Cl)Sc2ccccc21</smiles><smiles>[R1]N1/C(=C/C=C2\CC[Z17]([IH2+])C(/C=C/C3=[N+]([R2])c4ccc(C(=O)O)cc4C3(C)C)=C2Cl)C(C)(C)c2c1ccc1ccccc21</smiles>

FIgURE 1: Synthesized unsymmetrical heptamethine dyes with one carboxylic acid anchor group. $\mathrm{R}_{1}=$ ethyl, $\mathrm{R}_{2}=$ octyl. aggregation during immobilization on the semiconductor, the heterocycles were substituted with long alkyl chains.

\section{Materials and Methods}

2.1. General Information. Solvents and other chemicals were purchased from commercial sources (Aldrich, VWR, FEW, ABCR, and Solaronix) and used without purification. Unless otherwise stated, all reactions were carried out under inert gas (Argon) in anhydrous solvent. Sodium acetate was dried under high vacuum at $120^{\circ} \mathrm{C}$ for $3 \mathrm{~h} .{ }^{1} \mathrm{H}$ and ${ }^{13} \mathrm{C} \mathrm{NMR}$ spectra were recorded at $298 \mathrm{~K}$ on a $5 \mathrm{~mm}$ broadband inverse probe using a Bruker Avance 400 NMR spectrometer at 400.1 and $100.6 \mathrm{MHz}$, respectively. Chemical shifts $(\delta)$ in ppm are calibrated to residual solvent peaks (DMSO: $\delta=2.49$ and $39.5 \mathrm{ppm}$, resp.). Coupling constants $J$ are reported in $\mathrm{Hz}$ and for ${ }^{1} \mathrm{H}$ NMR data coupling patterns are described as $\mathrm{s}=$ singlet, $\mathrm{d}=$ doublet, $\mathrm{t}=$ triplet, $\mathrm{q}=$ quartet, $\mathrm{m}=$ multiplet, and br = broad and for ${ }^{13} \mathrm{C}$ NMR data as $\mathrm{s}=$ quaternary carbon, $\mathrm{d}=\mathrm{CH}, \mathrm{t}=\mathrm{CH}_{2}$, and $\mathrm{q}=\mathrm{CH}_{3}$. For the $1 \mathrm{D}$ and $2 \mathrm{D}$ NMR experiments standard Bruker pulse programs and measuring parameters were applied. Double quantum filtered homonuclear correlation spectroscopy (DQF-COSY), gradient selected heteronuclear single quantum coherence (gHSQC), and heteronuclear multiple bond coherence (gHMBC) were used for ${ }^{1} \mathrm{H}$ and ${ }^{13} \mathrm{C}$ NMR chemical shift assignments ( $w=$ weak correlation peak). Some of the compounds were only poorly soluble in DMSO and, therefore, the signal to noise of the $1 \mathrm{D}{ }^{13} \mathrm{C}$ NMR spectra was rather low (S15, Hepta3, Hepta4, Hepta5, and Hepta6). In some cases, resonances of quaternary carbons were missing in these spectra ( $\mathbf{S 1 1}$ and S13) or only 2D correlated NMR data was recorded (Heptal and Hepta7). Copies of all spectra are provided in the Supplementary Material available online at http://dx.doi.org/10.1155/2014/258984. MS data were recorded on a Bruker maXis-ESI-Q-TOF mass spectrometer (ESI-QTOF) or on a HiRes-ESI IonSpec Varian Ultima-ESI FTICR-MS spectrometer (USA). Elemental analyses for C, H, $\mathrm{N}$, and $\mathrm{O}$ were measured by an apparatus from LECO. The elements $\mathrm{Cl}, \mathrm{Br}$, and I were determined by titration or with ion chromatograph after Schoeniger digestion. IR spectra were recorded on a Bruker Tensor 27 with ATR configuration. UVvis spectra of the dyes in solutions were recorded on a Varian Cary 50 Scan spectrometer in a $1.0 \mathrm{~cm}$ square cuvette. Cyclic voltammetry measurements were performed on a PGStat 30 potentiostat (Autolab) using a three-cell electrode system consisting of a rotating gold working electrode, platinum counter electrode, and $\mathrm{Ag} / \mathrm{AgCl}$ (0.1 M tetrabutylammonium chloride in anhydrous DMF or acetonitrile) reference electrode. Thermogravimetric analysis (TGA) was performed on a Netzsch TG 209 F1 under nitrogen atmosphere.

2.2. Synthesis. More information about the precursors S1-S8, a detailed description of the synthesis, and characterization of the precursors and half dyes S9-S16 is given in the Supplementary Material. 
2.2.1. 2-(2-(3-(2-(6-Carboxy-1-ethylquinolin-2(1H)-ylidene) ethylidene)-2-chlorocyclohex-1-en-1-yl)vinyl)-3-ethyl-1,1dimethyl-1H-benzo[e]indol-3-ium Iodide (Hepta1). Half dye S11 (1.54 mmol, $0.92 \mathrm{~g}$ ) was dissolved in anhydrous ethanol (40 mL). Subsequently, 6-carboxy-1-ethyl-2-methylquinolin1-ium bromide S10 (1.54 mmol, $0.46 \mathrm{~g}$ ) and sodium acetate were added to the solution at room temperature. Then, the reaction mixture was heated to reflux for $1 \mathrm{~h}$, and the product started to precipitate. In order to dilute the thick suspension, ethanol $(40 \mathrm{~mL})$ was added and heating was continued for $15 \mathrm{~h}$ under vigorous stirring. Finally, the suspension was chilled to room temperature. The precipitate was collected by filtration, washed with cold ethanol three times $(50 \mathrm{~mL})$, and dried under reduced pressure at $40^{\circ} \mathrm{C}$ to yield the dye Heptal (0.77 g, 75\%). d.p. $223^{\circ}$ C. ${ }^{1} \mathrm{H}$ NMR (DMSO-d $6,400.1 \mathrm{MHz}$ ): $\delta 8.76(\mathrm{~d}, J=9.2,1 \mathrm{H}, \mathrm{H}-27), 8.66(\mathrm{~d}, J=1.9,1 \mathrm{H}, \mathrm{H}-33), 8.43$ (dd, $J=9.1,1.9,1 \mathrm{H}, \mathrm{H}-31), 8.36$ (d, $J=9.2,1 \mathrm{H}, \mathrm{H}-26), 8.36$ (d, $J=14.7,1 \mathrm{H}, \mathrm{H}-23), 8.30$ (d, $J=9.1,1 \mathrm{H}, \mathrm{H}-30), 8.16$ (d, $J=$ 8.6, 1H, H-3), 7.97 (d, J = 13.3, 1H, H-16), 7.94 (m, 1H, H-6), $7.94(\mathrm{~d}, J=8.8,1 \mathrm{H}, \mathrm{H}-7), 7.53(\mathrm{~m}, 1 \mathrm{H}, \mathrm{H}-4), 7.50$ (d, $J=8.8$, $1 \mathrm{H}, \mathrm{H}-8), 7.35$ (m, 1H, H-5), 6.86 (d, $J=14.7,1 \mathrm{H}, \mathrm{H}-24), 5.87$ (d, $J=13.3,1 \mathrm{H}, \mathrm{H}-15), 4.91$ (q, $J=7.1,2 \mathrm{H}, \mathrm{H}-37), 4.08$ (q, $J=$ 7.0, 2H, H-35), 2.77 (t, $J=5.9,2 \mathrm{H}, \mathrm{H}-20), 2.69$ (t, $J=5.9,2 \mathrm{H}$, $\mathrm{H}-18), 1.91$ (s, 6H, H-13, 14), 1.88 (m, 2H, H-19), 1.53 (t, $J=7.1$, $3 \mathrm{H}, \mathrm{H}-38$ ), 1.26 (t, $J=7.0,3 \mathrm{H}, \mathrm{H}-36) .{ }^{13} \mathrm{C}$ NMR (DMSO- $\mathrm{d}_{6}$, 100.6 MHz): $\delta 166.0$ (s, C-34), 165.3 (s, C-12), 153.8 (s, C-25), 143.5 (d, C-23), 143.3 (s, C-22), 142.1 (d, C-27), 140.4 (s, C-9), 139.4 (s, C-29), 135.6 (s, C-32), 134.6 (d, C-31), 134.0 (d, C-16), 130.4 (d, C-33), 130.1 (s, C-10), 13.2 (q, C-38), 129.9 (s, C-1), 129.7 (d, C-7), 129.7 (d, C-6), 128.1 (s, C-2), 127.3 (s, C-21), 127.1 (d, C-4), 126.7 (s, C-28), 124.1 (s, C-17), 123.2 (d, C-5), 121.6 (d, C-3), 120.7 (d, C-26), 117.5 (d, C-30), 113.0 (d, C-24), 110.3 (d, C-8), 94.8 (d, C-15), 48.6 (s, C-11), 45.5 (t, C-37), 37.3 (t, C-35), 26.9 (q, C-13, 14), 26.6 (t, C-20), 25.6 (t, C-18), 20.6 (t, C-19), 11.7 (q, C-36). HMBC correlations: $\mathrm{H}-3 \rightarrow$ $\mathrm{C}-(1,2 \mathrm{w}, 5,10), \mathrm{H}-4 \rightarrow \mathrm{C}-(2,6), \mathrm{H}-5 \rightarrow \mathrm{C}-(1,3), \mathrm{H}-6 \rightarrow$ $\mathrm{C}-(1 \mathrm{w}, 2,4,7), \mathrm{H}-7 \rightarrow \mathrm{C}-(1 \mathrm{w}, 2,6,9), \mathrm{H}-8 \rightarrow \mathrm{C}-(1,10), \mathrm{H}-13$, $14 \rightarrow \mathrm{C}-(10,11,12,13,14), \mathrm{H}-15 \rightarrow \mathrm{C}-(11,12 \mathrm{w}, 17), \mathrm{H}-16 \rightarrow$ $\mathrm{C}-(12,18,22), \mathrm{H}-18 \rightarrow \mathrm{C}-(19,20,21 w, 22), \mathrm{H}-19 \rightarrow \mathrm{C}-(17$, $21), \mathrm{H}-20 \rightarrow \mathrm{C}-(17 \mathrm{w}, 18,19,22), \mathrm{H}-23 \rightarrow \mathrm{C}-(20,22,25)$, $\mathrm{H}-24 \rightarrow \mathrm{C}-(21,23 \mathrm{w}, 26), \mathrm{H}-26 \rightarrow \mathrm{C}-(24 \mathrm{w}, 28), \mathrm{H}-27 \rightarrow$ C- $(25,29,33)$, H-30 $\rightarrow$ C-(28, 31w, 32), H-31 $\rightarrow$ C- $(29,33$, $34)$, H-33 $\rightarrow$ C-(27, 28w, 29, 31, 34), H-35 $\rightarrow$ C- $(9,12,36)$, $\mathrm{H}-36 \rightarrow \mathrm{C}-(35), \mathrm{H}-37 \rightarrow \mathrm{C}-(25,29,38), \mathrm{H}-38 \rightarrow \mathrm{C}-(37)$. DQF-COSY correlations: $\mathrm{H}-3 \rightarrow \mathrm{H}-(4), \mathrm{H}-4 \rightarrow \mathrm{H}-(3,5)$, $\mathrm{H}-5 \rightarrow \mathrm{H}-(4,6), \mathrm{H}-6 \rightarrow \mathrm{H}-(5), \mathrm{H}-7 \rightarrow \mathrm{H}-(8), \mathrm{H}-8 \rightarrow$ $\mathrm{H}-(7), \mathrm{H}-15 \rightarrow \mathrm{H}-(16), \mathrm{H}-16 \rightarrow \mathrm{H}-(15), \mathrm{H}-18 \rightarrow \mathrm{H}-(19)$, $\mathrm{H}-19 \rightarrow \mathrm{H}-(18,20), \mathrm{H}-20 \rightarrow \mathrm{H}-(19), \mathrm{H}-23 \rightarrow \mathrm{H}-(24)$, $\mathrm{H}-24 \rightarrow \mathrm{H}-(23), \mathrm{H}-26 \rightarrow \mathrm{H}-(27), \mathrm{H}-27 \rightarrow \mathrm{H}-(26), \mathrm{H}-30$ $\rightarrow \mathrm{H}-(31), \mathrm{H}-31 \rightarrow \mathrm{H}-(30,33), \mathrm{H}-33 \rightarrow \mathrm{H}-(31), \mathrm{H}-35 \rightarrow$ $\mathrm{H}-(36), \mathrm{H}-36 \rightarrow \mathrm{H}-(35), \mathrm{H}-37 \rightarrow \mathrm{H}-(38), \mathrm{H}-38 \rightarrow \mathrm{H}-(37)$. HR-MS (ESI-QTOF): calculated for $\mathrm{C}_{38} \mathrm{H}_{38} \mathrm{ClN}_{2} \mathrm{O}_{2}[\mathrm{M}-\mathrm{I}]^{+}$: 589.2616. Found: 589.2611. Elemental analysis: calculated for $\mathrm{C}_{38} \mathrm{H}_{38} \mathrm{~N}_{2} \mathrm{O}_{2}$ ClI: C, 63.65\%; H, 5.34\%; N, 3.91\%; O, 4.46\%; Cl, 4.94\%; I, 17.70\%. Found: C, 65.58\%; H, 5.88\%; N, 4.98\%. IR (ATR, $\left.\mathrm{cm}^{-1}\right): \lambda_{\max } 3397,2975,2932,2868,1701,1621,1551$, 1429, 1234, 1146, 1031, 995.
2.2.2. 2-(2-(3-(2-(6-Carboxy-1-ethylquinolin-2(1H)-ylidene) ethylidene)-2-chlorocyclohex-1-en-1-yl)vinyl)-1,1-dimethyl-3octyl-1H-benzo[e]indol-3-ium Iodide (Hepta2). Half dye S13 (4.41 mmol, $3.00 \mathrm{~g}$ ), 6-carboxy-1-ethyl-2-methylquinolinium bromide $\mathbf{S 1 0}(4.41 \mathrm{mmol}, 1.30 \mathrm{~g})$, and sodium acetate $(8.8 \mathrm{mmol}, 0.72 \mathrm{~g})$ were suspended in ethanol $(120 \mathrm{~mL})$. The reaction mixture was heated to reflux for $18 \mathrm{~h}$ and subsequently cooled to room temperature. The precipitate was isolated by filtration, washed with cold ethanol twice $(50 \mathrm{~mL})$, and dried at $40^{\circ} \mathrm{C}$ under reduced pressure. For further purification, the product was recrystallized from methanol to yield brown crystals of Hepta2 (1.98 g, 56\%). d.p. $206^{\circ} \mathrm{C},{ }^{1} \mathrm{H}$ NMR (DMSO-d $\left.6,400.1 \mathrm{MHz}\right): \delta 8.78(\mathrm{~d}, J=$ 9.2, $1 \mathrm{H}, \mathrm{H}-27), 8.63$ (d, $J=1.6,1 \mathrm{H}, \mathrm{H}-33), 8.44$ (dd, $J=9.2$, 1.6, $1 \mathrm{H}, \mathrm{H}-31$ ), 8.34 (d, $J=9.2,1 \mathrm{H}, \mathrm{H}-26), 8.32$ (d, $J=14.6$, $1 \mathrm{H}, \mathrm{H}-23$ ), 8.29 (d, $J=9.2,1 \mathrm{H}, \mathrm{H}-30), 8.14$ (d, $J=8.7,1 \mathrm{H}$, H-6), 7.92 (m, 1H, H-3), 7.91 (d, $J=13.3,1 \mathrm{H}, \mathrm{H}-16), 7.90$ (m, 1H, H-7), 7.53 (m, 1H, H-5), 7.48 (d, $J=8.9,1 \mathrm{H}, \mathrm{H}-8), 7.34$ (m, 1H, H-4), 6.89 (d, $J=14.6,1 \mathrm{H}, \mathrm{H}-24), 5.82$ (d, $J=13.3$, $1 \mathrm{H}, \mathrm{H}-15), 4.93$ (q (br), $J=7,2 \mathrm{H}, \mathrm{H}-43), 4.00$ (m, 2H, H-35), $2.76(\mathrm{t}, J=5.0,2 \mathrm{H}, \mathrm{H}-20), 2.66(\mathrm{t}, J=5.0,2 \mathrm{H}, \mathrm{H}-18), 1.89(\mathrm{~s}$, 6H, H-13, 14), 1.85 (m, 2H, H-19), 1.69 (m, 2H, H-36), 1.53 (t, $J=7,3 \mathrm{H}, \mathrm{H}-44), 1.37$ (m, 2H, H-37), 1.32 (m, 2H, H-38), 1.24 (m, 2H, H-39), 1.22 (m, 2H, H-41), 1.22 (m, 2H, H-40), 0.82 $(\mathrm{t}, J=6.9,3 \mathrm{H}, \mathrm{H}-42) .{ }^{13} \mathrm{C}$ NMR (DMSO-d $\left.6,100.6 \mathrm{MHz}\right)$ : $\delta 166.0$ (s, C-34), 165.3 (s, C-12), 153.9 (s, C-25), 143.4 (d, C-23), 143.0 (s, C-22), 142.5 (d, C-27), 141.0 (s, C-9), 139.1 (s, C-29), 137.4 (s, C-32), 135.0 (d, C-31), 133.4 (d, C-16), 130.3 (d, C-33), 130.1 (s, C-2), 129.8 (s, C-10), 129.8 (d, C-7), 129.8 (d, C-3), 128.1 (s, C-1), 127.5 (s, C-21), 127.2 (d, C-5), 126.9 (s, C-28), 124.0 (s, C-17), 123.2 (d, C-4), 121.6 (d, C-6), 120.6 (d, C-26), 117.3 (d, C-30), 113.6 (d, C-24), 110.6 (d, C-8), 94.8 (d, C-15), 48.6 (s, C-11), 45.7 (t, C-43), 42.3 (t, C-35), 31.2 (t, C-40), 28.7 (t, C-38), 28.6 (t, C-39), 27.1 (q, C-13, 14), 26.7 (t, C-20), 26.4 (t, C-36), 26.1 (t, C-37), 25.7 (t, C-18), 22.0 (t, C-41), 20.7 (t, C-19), 13.9 (q, C-42), 13.4 (q, C-44). HMBC correlations: H-3 $\rightarrow$ C- $(1,5,10), \mathrm{H}-4 \rightarrow \mathrm{C}-(2,5 \mathrm{w}, 6), \mathrm{H}-5$ $\rightarrow$ C- $(1,3,4 \mathrm{w}), \mathrm{H}-6 \rightarrow \mathrm{C}-(1 \mathrm{w}, 2,4,7), \mathrm{H}-7 \rightarrow \mathrm{C}-(1,6 \mathrm{w}$, 9), $\mathrm{H}-8 \rightarrow \mathrm{C}-(1,10), \mathrm{H}-13,14 \rightarrow \mathrm{C}-(10,11,12,13,14), \mathrm{H}-15$ $\rightarrow \mathrm{C}-(11,12,13$ \& 14w, 17), H-16 $\rightarrow$ C- $(12,18,22), \mathrm{H}-18 \rightarrow$ $\mathrm{C}-(17,19,20,22), \mathrm{H}-19 \rightarrow \mathrm{C}-(17,21), \mathrm{H}-20 \rightarrow \mathrm{C}-(18,19,21$, 22), $\mathrm{H}-23 \rightarrow \mathrm{C}-(20,22,25), \mathrm{H}-24 \rightarrow \mathrm{C}-(21,23 \mathrm{w}, 26), \mathrm{H}-26$ $\rightarrow$ C-(24w, 25, 28), H-27 $\rightarrow$ C- $(25,28 w, 29,33)$, H-30 $\rightarrow$ $\mathrm{C}-(28,32), \mathrm{H}-31 \rightarrow \mathrm{C}-(29,33,34), \mathrm{H}-33 \rightarrow \mathrm{C}-(27,28 \mathrm{w}, 29$, $31,34), \mathrm{H}-35 \rightarrow \mathrm{C}-(9,12,37), \mathrm{H}-36 \rightarrow \mathrm{C}-(37,38), \mathrm{H}-37$ $\rightarrow$ C-(39), H-40 $\rightarrow$ C-(42), H-42 $\rightarrow$ C-(40, 41), H-43 $\rightarrow$ C-(25, 29, 44). DQF-COSY correlations: H-3 $\rightarrow$ H-(4), $\mathrm{H}-4 \rightarrow \mathrm{H}-(3,5), \mathrm{H}-5 \rightarrow \mathrm{H}-(4,6), \mathrm{H}-6 \rightarrow \mathrm{H}-(5), \mathrm{H}-7 \rightarrow$ H-(8), H-8 $\rightarrow$ H-(7), H-15 $\rightarrow$ H-(16), H-16 $\rightarrow$ H-(15), H-18 $\rightarrow \mathrm{H}-(19), \mathrm{H}-19 \rightarrow \mathrm{H}-(18,20), \mathrm{H}-20 \rightarrow \mathrm{H}-(19), \mathrm{H}-23 \rightarrow$ $\mathrm{H}-(24), \mathrm{H}-24 \rightarrow \mathrm{H}-(23), \mathrm{H}-26 \rightarrow \mathrm{H}-(27), \mathrm{H}-27 \rightarrow \mathrm{H}-(26)$, $\mathrm{H}-30 \rightarrow \mathrm{H}-(31), \mathrm{H}-31 \rightarrow \mathrm{H}-(30,33), \mathrm{H}-33 \rightarrow \mathrm{H}-(31), \mathrm{H}-35$ $\rightarrow \mathrm{H}-(36), \mathrm{H}-36 \rightarrow \mathrm{H}-(35,37), \mathrm{H}-37 \rightarrow \mathrm{H}-(36), \mathrm{H}-41 \rightarrow$ $\mathrm{H}-(42), \mathrm{H}-42 \rightarrow \mathrm{H}-(41), \mathrm{H}-43 \rightarrow \mathrm{H}-(44), \mathrm{H}-44 \rightarrow \mathrm{H}-(43)$. HR-MS (ESI-QTOF): calculated for $\mathrm{C}_{44} \mathrm{H}_{50} \mathrm{ClN}_{2} \mathrm{O}_{2}[\mathrm{M}-\mathrm{I}]^{+}$: 673.3555. Found: 673.3546. Elemental analysis: calculated for $\mathrm{C}_{44} \mathrm{H}_{50} \mathrm{ClIN}_{2} \mathrm{O}_{2}$ : C, 65.96\%; H, 6.29\%; N, 3.50\%; O, 3.99\%; 
Cl, 4.42\%; I, 15.84\%. Found: C, 72.27\%; H, 6.73\%; N, 3.92\%; O, 5.62\%; Cl, 5.04\%; I, 5.15\%. IR (ATR, $\left.\mathrm{cm}^{-1}\right): \lambda_{\max } 2927$, 2856, 1704, 1617, 1540, 1430, 1342, 1224, 1039, 744.

2.2.3. 2-(2-(3-(2-(5-Carboxy-3,3-dimethyl-1-octylindolin2-ylidene)ethylidene)-2-chlorocyclohex-1-en-1-yl)vinyl)-1octylquinolin-1-ium Iodide (Hepta3). Half dye S12 (4.9 mmol, $3.00 \mathrm{~g}$ ), 5-carboxy-2,3,3-trimethyl-1-octyl-3H-indol1-ium iodide S8 (4.9 mmol, $2.16 \mathrm{~g}$ ), and sodium acetate (9.8 mmol, $0.8 \mathrm{~g})$ were suspended in ethanol $(120 \mathrm{~mL})$ and heated to reflux for $48 \mathrm{~h}$. Then, the suspension was cooled to room temperature and stored at $4^{\circ} \mathrm{C}$ overnight. The precipitate was collected by filtration. For further purification, the dye was recrystallized from methanol twice $(60 \mathrm{~mL})$ to yield green crystals of Hepta3 (0.61 g, 18\%). d.p. $244^{\circ} \mathrm{C}$. ${ }^{1} \mathrm{H}$ NMR (DMSO-d $\left.{ }_{6}, 400.1 \mathrm{MHz}\right): \delta 12.57(\mathrm{~s}, 1 \mathrm{H}$, $\mathrm{OH}), 8.85$ (d, $J=9.2,1 \mathrm{H}, \mathrm{H}-7), 8.45$ (d, $J=9.3,1 \mathrm{H}, \mathrm{H}-3), 8.45$ (d, $J=9.2,1 \mathrm{H}, \mathrm{H}-8), 8.31$ (d, $J=14.6,1 \mathrm{H}, \mathrm{H}-11), 8.29$ (dd, $J=$ 8.0, 1.2, 1H, H-6), 8.11 (m, 1H, H-4), 7.87 (s, 1H, H-29), 7.87 (m, 1H, H-5), 7.84 (d, $J=8.2,1 \mathrm{H}, \mathrm{H}-27), 7.69$ (d, $J=12.8, \mathrm{H}$, $\mathrm{H}-18$ ), 7.03 (d, $J=8.2,1 \mathrm{H}, \mathrm{H}-26), 7.02$ (d, $J=14.6,1 \mathrm{H}, \mathrm{H}-10$ ), $5.81(\mathrm{~d}, J=12.8,1 \mathrm{H}, \mathrm{H}-19), 4.94(\mathrm{t}, J=7.4,2 \mathrm{H}, \mathrm{H}-31), 3.85(\mathrm{t}$, $J=6.5,2 \mathrm{H}, \mathrm{H}-39), 2.74$ (m, 2H, H-13), 2.65 (m, 2H, H-15), 1.88 (m, 2H, H-32), 1.85 (m, 2H, H-14), 1.62 (m, 2H, H-40), 1.60 (s, 6H, H-22, 23), 1.52 (m, 2H, H-33), 1.35 (m, 2H, H-34), 1.33 (m, 2H, H-41), 1.25 (m, 4H, H-37, 45), 1.23 (m, 4H, H-36, 44), 1.2-1.3 (m, 6H, H-35, 42, 43), 0.83, 0.85 (m, 6H, H-38, 46). ${ }^{13} \mathrm{C}$ NMR (DMSO- $\left.\mathrm{d}_{6}, 100.6 \mathrm{MHz}\right): \delta 167.2$ (s, C-30), 161.5 (s, C-20), 154.7 (s, C-9), 147.5 (s, C-25), 143.5 (d, C-11), 143.1 (d, C-7), 141.9 (s, C-17), 139.0 (s, C-24), 138.5 (s, C-2), 134.8 (d, C-4), 131.2 (d, C-18), 130.7 (d, C-27), 130.2 (d, C-6), 128.6 (s, C-12), 128.5 (d, C-5), 127.6 (s, C-1), 125.3 (s, C-16), 123.0 (d, C-29), 122.9 (s, C-28), 121.2 (d, C-8), 118.7 (d, C-3), 116.2 (d, C-10), 107.5 (d, C-26), 95.8 (d, C-19), 50.0 (t, C-31), 45.8 (s, C-21), 41.9 (t, C-39), 31.2 (t, C-36, 44), 28.6 (t, C-34), 28.4-28.6 (t, C-35, 42, 43), 28.1 (t, C-32), 27.8 (q, C-22, 23), 26.8 (t, C-13), 26.1 (t, C-41), 25.9 (t, C-40), 25.7 (t, C-33), 25.7 (t, C-15), 22.0 (t, C-37, 45), 20.8 (t, C-14), 13.9, 14.0 (q, C-38, 46). HMBC correlations: $\mathrm{H}-3 \rightarrow \mathrm{C}-(1,5), \mathrm{H}-4 \rightarrow \mathrm{C}-(2,6)$, $\mathrm{H}-5 \rightarrow$ C- $(1,3,6), \mathrm{H}-6 \rightarrow \mathrm{C}-(1 \mathrm{w}, 2,4,7), \mathrm{H}-7 \rightarrow \mathrm{C}-(2,6$, 9), $\mathrm{H}-8 \rightarrow \mathrm{C}-(9,10 \mathrm{w}), \mathrm{H}-10 \rightarrow \mathrm{C}-(8,12), \mathrm{H}-11 \rightarrow \mathrm{C}-(9,13$, $17), \mathrm{H}-13 \rightarrow \mathrm{C}-(12,17), \mathrm{H}-15 \rightarrow \mathrm{C}-(16), \mathrm{H}-18 \rightarrow \mathrm{C}-(15,17)$, $\mathrm{H}-19 \rightarrow \mathrm{C}-(21), \mathrm{H}-22,23 \rightarrow \mathrm{C}-(20,21,22,23,24), \mathrm{H}-26 \rightarrow$ $\mathrm{C}-(24,28), \mathrm{H}-27 \rightarrow \mathrm{C}-(25,29,30), \mathrm{H}-29 \rightarrow \mathrm{C}-(21,25,30)$, $\mathrm{H}-31 \rightarrow \mathrm{C}-(2,9 \mathrm{w}), \mathrm{H}-35,42,43 \rightarrow \mathrm{C}-(36,44), \mathrm{H}-38,46 \rightarrow$ $\mathrm{C}-(36,44,37,45)$. DQF-COSY correlations: H-3 $\rightarrow \mathrm{H}-(4)$, $\mathrm{H}-4 \rightarrow \mathrm{H}-(3,5), \mathrm{H}-5 \rightarrow \mathrm{H}-(4,6), \mathrm{H}-6 \rightarrow \mathrm{H}-(5), \mathrm{H}-7 \rightarrow$ $\mathrm{H}-(7), \mathrm{H}-8 \rightarrow \mathrm{H}-(8), \mathrm{H}-10 \rightarrow \mathrm{H}-(11), \mathrm{H}-11 \rightarrow \mathrm{H}-(10), \mathrm{H}-13$ $\rightarrow \mathrm{H}-(14), \mathrm{H}-14 \rightarrow \mathrm{H}-(13,15), \mathrm{H}-15 \rightarrow \mathrm{H}-(14), \mathrm{H}-18 \rightarrow$ $\mathrm{H}-(19), \mathrm{H}-19 \rightarrow \mathrm{H}-(18), \mathrm{H}-26 \rightarrow \mathrm{H}-(27), \mathrm{H}-27 \rightarrow \mathrm{H}-(26)$, $\mathrm{H}-31 \rightarrow \mathrm{H}-(32), \mathrm{H}-32 \rightarrow \mathrm{H}-(31,33), \mathrm{H}-33 \rightarrow \mathrm{H}-(32,34)$, $\mathrm{H}-34 \rightarrow \mathrm{H}-(33), \mathrm{H}-37,45 \rightarrow \mathrm{H}-(38,46), \mathrm{H}-38,46 \rightarrow \mathrm{H}-(37$, $45), \mathrm{H}-39 \rightarrow \mathrm{H}-(40), \mathrm{H}-40 \rightarrow \mathrm{H}-(39,41), \mathrm{H}-41 \rightarrow \mathrm{H}-(40)$. HR-MS (ESI-QTOF): calculated for $\mathrm{C}_{46} \mathrm{H}_{60} \mathrm{ClN}_{2} \mathrm{O}_{2}[\mathrm{M}-\mathrm{I}]^{+}$: 707.4338. Found: 707.4334. Elemental analysis: calculated for $\mathrm{C}_{46} \mathrm{H}_{60} \mathrm{ClIN}_{2} \mathrm{O}_{2}$ : C, 66.14\%; H, 7.24\%; N, 3.35\%; O, 3.83\%; Cl, 4.24\%; I, 15.19\%. Found: C, 66.15\%; H, 7.29\%; N, 3.32\%;
O, 3.92\%; Cl, 4.03\%; I, 14.89\%. IR (ATR, $\left.\mathrm{cm}^{-1}\right): \lambda_{\max } 2923$, $2854,1708,1663,1600,1566,1453,1270,1120,935,772$.

2.2.4. 2-(2-(6-(2-(5-Carboxy-3,3-dimethyl-1-octylindolin2-ylidene)ethylidene)-3,4,5,6-tetrahydro-[1,1'-biphenyl]-2-

yl)vinyl)-1-octylquinolin-1-ium Iodide (Hepta4). Half dye S16 (3.8 mmol, 2.50 g), 5-carboxy-2,3,3-trimethyl-1-octyl$3 \mathrm{H}$-indol-1-ium iodide S8 $(3.8 \mathrm{mmol}, 1.69 \mathrm{~g})$, and sodium acetate $(8.9 \mathrm{mmol}, 0.6 \mathrm{~g})$ were suspended in ethanol $(60 \mathrm{~mL})$ and heated to reflux for $48 \mathrm{~h}$. Then, the suspension was cooled to room temperature and stored at $4^{\circ} \mathrm{C}$ for $12 \mathrm{~h}$. Subsequently, the precipitated starting material was removed by filtration. The volume of the filtrate was reduced to half under reduced pressure and stored at $4^{\circ} \mathrm{C}$ for $20 \mathrm{~h}$. Finally, a fine crystalline powder precipitated, which was collected by filtration. The crude product was further purified by recrystallization from ethanol to yield the pure dye Hepta4 (0.42 g, $12.6 \%$ ). m.p. $160^{\circ}$ C. d.p. $220^{\circ}$ C. ${ }^{1} \mathrm{H}$ NMR (DMSO- $\mathrm{d}_{6}$, $400.1 \mathrm{MHz}$ ): $\delta 12.50$ (s, H, OH), 8.60 (d, $J=9.1,1 \mathrm{H}, \mathrm{H}-7)$, $8.34(\mathrm{~d}, J=9.1,1 \mathrm{H}, \mathrm{H}-3), 8.12(\mathrm{~d}, J=8.0,1 \mathrm{H}, \mathrm{H}-6), 8.04(\mathrm{~m}$, $1 \mathrm{H}, \mathrm{H}-4), 7.78$ (d, $J=8.3,1 \mathrm{H}, \mathrm{H}-27), 7.76$ (m, 1H, H-5), 7.69 (s, 1H, H-29), 7.56 (m, 2H, H-49, 51), 7.54 (m, 1H, H-50), 7.45 (d, $J=9.1,1 \mathrm{H}, \mathrm{H}-8), 7.32$ (d, $J=14.7,1 \mathrm{H}, \mathrm{H}-11), 7.21$ (d, $J=7.7,2 \mathrm{H}, \mathrm{H}-48,52), 6.96(\mathrm{~d}, J=8.3,1 \mathrm{H}, \mathrm{H}-26), 6.82(\mathrm{~d}$, $J=14.7,1 \mathrm{H}, \mathrm{H}-10), 6.42$ (d, $J=12.9,1 \mathrm{H}, \mathrm{H}-18), 5.78$ (d, $J=$ 12.9, 1H, H-19), 4.82 (m, 2H, H-31), 3.79 (m, 2H, H-39), 2.72 (m, 2H, H-13), 2.63 (m, 2H, H-15), 1.95 (m, 2H, H-14), 1.83 (m, 2H, H-32), 1.58 (m, 2H, H-40), 1.53 (m, 2H, H-33), 1.39 (m, 2H, H-34), 1.31 (m, 2H, H-41), 1.25 (m, 4H, H-37, 45), 1.23 (m, 4H, H-36, 44), 1.2-1.4 (m, 6H, H-35, 42, 43), 1.05 (s, $6 \mathrm{H}, \mathrm{H}-22,23), 0.84,0.87(\mathrm{t}, J=7.0,6 \mathrm{H}, \mathrm{H}-38,46) .{ }^{13} \mathrm{C} \mathrm{NMR}$ $\left(\mathrm{DMSO}_{\mathrm{d}}, 100.6 \mathrm{MHz}\right): \delta 167.1$ (s, C-30), 160.5 (s, C-20), 154.5 (s, C-17), 154.3 (s, C-9), 147.5 (s, C-25), 146.6 (d, C-11), 142.4 (d, C-7), 138.7 (s, C-24), 138.4 (s, C-2), 138.3 (s, C-47), 135.0 (d, C-18), 134.6 (d, C-4), 130.9 (s, C-16), 130.6 (s, C-12), 130.6 (d, C-27), 130.1 (d, C-6), 129.8 (d, C-48, 52), 128.3 (d, C-49, 51), 128.0 (d, C-5), 127.8 (d, C-50), 126.9 (s, C-1), 122.7 (s, C-28), 122.7 (d, C-29), 119.6 (d, C-8), 118.3 (d, C-3), 112.5 (d, C-10), 107.3 (d, C-26), 96.1 (d, C-19), 49.7 (t, C-31), 45.2 (s, C-21), 41.9 (t, C-39), 31.2 (t, C-36, 44), 28.5 (t, C-34), 28.4-28.6 (t, C-35, 42, 43), 27.9 (t, C-32), 27.3 (q, C-22, 23), 26.1 (t, C-41), 25.8 (t, C-40), 25.6 (t, C-33), 25.1 (t, C-13), 24.3 (t, C-15), 22.0 (t, C-37, 45), 21.2 (t, C-14), 13.9, 14.0 (q, C-38, 46). HMBC correlations: $\mathrm{H}-3 \rightarrow \mathrm{C}-(1,5), \mathrm{H}-4 \rightarrow \mathrm{C}-(2,6)$, $\mathrm{H}-5 \rightarrow \mathrm{C}-(1,3,4 \mathrm{w}, 6 \mathrm{w}), \mathrm{H}-6 \rightarrow \mathrm{C}-(1 \mathrm{w}, 2,4,7), \mathrm{H}-7 \rightarrow$ $\mathrm{C}-(1 \mathrm{w}, 2,6,9), \mathrm{H}-8 \rightarrow \mathrm{C}-(1,9,10 \mathrm{w}), \mathrm{H}-10 \rightarrow \mathrm{C}-(8,11 \mathrm{w}, 12)$, $\mathrm{H}-11 \rightarrow \mathrm{C}-(9,13), \mathrm{H}-13 \rightarrow \mathrm{C}-(12,14,15,17), \mathrm{H}-14 \rightarrow \mathrm{C}-(12$, $16), \mathrm{H}-15 \rightarrow \mathrm{C}-(13,14,16,17), \mathrm{H}-18 \rightarrow \mathrm{C}-(15,17,20), \mathrm{H}-19$ $\rightarrow \mathrm{C}-(16,20,21), \mathrm{H}-22,23 \rightarrow \mathrm{C}-(20,21,22,23,24), \mathrm{H}-26 \rightarrow$ $\mathrm{C}-(24,28), \mathrm{H}-27 \rightarrow \mathrm{C}-(25,29,30), \mathrm{H}-29 \rightarrow \mathrm{C}-(21,25,27$, $30), \mathrm{H}-31 \rightarrow \mathrm{C}-(2,9,32), \mathrm{H}-32 \rightarrow \mathrm{C}-(31), \mathrm{H}-33 \rightarrow \mathrm{C}-(31$, $32,35), \mathrm{H}-35,42,43 \rightarrow \mathrm{C}-(36,44), \mathrm{H}-36,44 \rightarrow \mathrm{C}-(38,46)$, $\mathrm{H}-38,46 \rightarrow \mathrm{C}-(36,44,37,38), \mathrm{H}-39 \rightarrow \mathrm{C}-(20,25,41), \mathrm{H}-40$ $\rightarrow \mathrm{C}-(41,42), \mathrm{H}-48,52 \rightarrow \mathrm{C}-(17,48,52,50), \mathrm{H}-49,51 \rightarrow$ C-(47, 49, 51), H-50 $\rightarrow$ C- $(48,52)$. DQF-COSY correlations: $\mathrm{H}-3 \rightarrow \mathrm{H}-(4), \mathrm{H}-4 \rightarrow \mathrm{H}-(3,5), \mathrm{H}-5 \rightarrow \mathrm{H}-(4,6), \mathrm{H}-6 \rightarrow$ $\mathrm{H}-(5), \mathrm{H}-7 \rightarrow \mathrm{H}-(8), \mathrm{H}-8 \rightarrow \mathrm{H}-(7), \mathrm{H}-10 \rightarrow \mathrm{H}-(11), \mathrm{H}-11$ 
$\rightarrow \mathrm{H}-(10), \mathrm{H}-13 \rightarrow \mathrm{H}-(14), \mathrm{H}-14 \rightarrow \mathrm{H}-(13,15), \mathrm{H}-15 \rightarrow$ $\mathrm{H}-(14), \mathrm{H}-18 \rightarrow \mathrm{H}-(19), \mathrm{H}-19 \rightarrow \mathrm{H}-(18), \mathrm{H}-26 \rightarrow \mathrm{H}-(27)$, $\mathrm{H}-27 \rightarrow \mathrm{H}-(26,29 \mathrm{w}), \mathrm{H}-29 \rightarrow \mathrm{H}-(27 \mathrm{w}), \mathrm{H}-31 \rightarrow \mathrm{H}-(32)$, $\mathrm{H}-32 \rightarrow \mathrm{H}-(31,33), \mathrm{H}-33 \rightarrow \mathrm{H}-(32), \mathrm{H}-39 \rightarrow \mathrm{H}-(40), \mathrm{H}-40$ $\rightarrow \mathrm{H}-(39,41), \mathrm{H}-41 \rightarrow \mathrm{H}-(40), \mathrm{H}-48,52 \rightarrow \mathrm{H}-(49,51)$, $\mathrm{H}-49,51 \rightarrow \mathrm{H}-(48,52)$. HR-MS (ESI-QTOF): calculated for $\mathrm{C}_{52} \mathrm{H}_{65} \mathrm{~N}_{2} \mathrm{O}_{2}$ [M-I] ${ }^{+}$: 749.5041. Found: 749.5039. Elemental analysis: calculated for $\mathrm{C}_{52} \mathrm{H}_{65} \mathrm{IN}_{2} \mathrm{O}_{2}$ : C, $71.22 \%$; $\mathrm{H}, 7.47 \%$; N, 3.19\%; O, 3.65\%; I, 14.47\%. Found: C, 70.82\%; H, 7.60\%; N, 3.17\%; O, 4.03\%; I, 14.23\%. IR (ATR, $\mathrm{cm}^{-1}$ ): $\lambda_{\max } 2924,2854$, 1707, 1669, 1453, 1143, 1104, 1049, 993, 745.

2.2.5. 4-(2-(3-(2-(5-Carboxy-3,3-dimethyl-1-octylindolin2-ylidene)ethylidene)-2-chlorocyclohex-1-en-1-yl)vinyl)-1octylquinolin-1-ium Perchlorate (Hepta5). Half dye S14 (6.52 mmol, $4.00 \mathrm{~g})$, 5-carboxy-2,3,3-trimethyl-1-octyl-3Hindol-1-ium iodide S8 $(6.52 \mathrm{mmol}, 2.89 \mathrm{~g})$, and sodium acetate $(13.04 \mathrm{mmol}, 1.08 \mathrm{~g})$ were suspended in ethanol $(160 \mathrm{~mL})$ and heated to reflux for $96 \mathrm{~h}$. Then, the suspension was cooled to room temperature. Precipitated half dye was separated by filtration. The volume of the filtrate was concentrated to half under reduced pressure and, subsequently, slowly dropped to a warm $\left(60^{\circ} \mathrm{C}\right)$ ethanolic sodium perchlorate solution $(50 \mathrm{~mL}, 7 \mathrm{wt} \%)$. In order to complete ion exchange, the solution was stirred and heated for $1 \mathrm{~h}$. Finally, the solution was slowly cooled to room temperature and stored at $4^{\circ} \mathrm{C}$ for $5 \mathrm{~d}$. Precipitated crystals were isolated by filtration, washed with ethanol twice $(20 \mathrm{~mL}$ each), and dried under reduced pressure at $40^{\circ} \mathrm{C}$ to yield the dye Hepta5 (0.45 g, 8.5\%). d.p. $246^{\circ}$ C. ${ }^{1} \mathrm{H}$ NMR (DMSO-d 6 , 400.1 MHz): $\delta 12.52(\mathrm{~s}, 1 \mathrm{H}, \mathrm{OH}), 9.14(\mathrm{~d}, J=6.7,1 \mathrm{H}, \mathrm{H}-7)$, 8.88 (dd, $J=8.9,1.1,1 \mathrm{H}, \mathrm{H}-5), 8.46$ (d, $J=9.1,1 \mathrm{H}, \mathrm{H}-2), 8.31$ $(\mathrm{d}, J=6.7,1 \mathrm{H}, \mathrm{H}-8), 8.28$ (d, $J=15.4,1 \mathrm{H}, \mathrm{H}-11), 8.19(\mathrm{~m}, 1 \mathrm{H}$, H-3), 7.95 (m, 1H, H-4), 7.85 (d, $J=1.6,1 \mathrm{H}, \mathrm{H}-29), 7.83$ (dd, $J=8.2,1.6,1 \mathrm{H}, \mathrm{H}-27), 7.63$ (d, $J=15.4,1 \mathrm{H}, \mathrm{H}-10), 7.61$ (d, $J=$ 12.8, 1H, H-18), 6.99 (d, $J=8.2,1 \mathrm{H}, \mathrm{H}-26), 5.77$ (d, $J=12.8$, 1H, H-19), 4.89 (t, J = 7.3, 2H, H-31), 3.82 (m, 2H, H-39), 2.81 (m, 2H, H-13), 2.64 (m, 2H, H-15), 1.91 (m, 2H, H-32), 1.85 (m, 2H, H-14), 1.62 (m, 2H, H-40), 1.60 (s, 6H, H-22, 23), 1.38 (m, 2H, H-33), 1.33 (m, 2H, H-41), 1.27 (m, 2H, H-34), 1.25 (m, 4H, H-37, 45), 1.23 (m, 4H, H-36, 44), 1.2-1.4 (m, $6 \mathrm{H}, \mathrm{H}-35,42,43), 0.84$ (t, $J=7,6 \mathrm{H}, \mathrm{H}-38,46),{ }^{13} \mathrm{C} \mathrm{NMR}$ $\left(\mathrm{DMSO}_{\mathrm{d}}, 100.6 \mathrm{MHz}\right): \delta 167.2$ (s, C-30), 160.1 (s, C-20), 152.2 (s, C-9), 147.7 (s, C-25), 146.7 (d, C-7), 139.9 (s, C-17), 139.9 (d, C-11), 138.8 (s, C-24), 137.8 (s, C-1), 134.8 (d, C-3), 130.7 (d, C-27), 129.5 (s, C-12), 129.4 (d, C-18), 128.9 (d, C-4), 126.5 (s, C-6), 126.5 (d, C-5), 125.6 (s, C-16), 122.9 (d, C-29), 122.6 (s, C-28), 119.0 (d, C-2), 118.9 (d, C-10), 115.6 (d, C-8), 107.1 (d, C-26), 95.5 (d, C-19), 56.3 (t, C-31), 45.5 (s, C-21), 41.8 (t, C-39), 31.2 (t, C-36, 44), 29.3 (t, C-32), 28.5 (t, C-34), 28.4-28.6 (t, C-35, 42, 43), 27.8 (q, C-22, 23), 27.0 (t, C-13), $26.1(\mathrm{t}, \mathrm{C}-41), 25.8(\mathrm{t}, \mathrm{C}-40), 25.8(\mathrm{t}, \mathrm{C}-33), 25.8(\mathrm{t}, \mathrm{C}-15)$, 22.0 (t, C-37, 45), 20.8 (t, C-14), 13.9 (q, C-38, 46). HMBC correlations: $\mathrm{H}-2 \rightarrow \mathrm{C}-(4,6), \mathrm{H}-3 \rightarrow \mathrm{C}-(1,2 \mathrm{w}, 5), \mathrm{H}-4 \rightarrow$ $\mathrm{C}-(2,3 \mathrm{w}, 6), \mathrm{H}-5 \rightarrow \mathrm{C}-(1,3,4 \mathrm{w}, 9), \mathrm{H}-7 \rightarrow \mathrm{C}-(1,8,9,31)$, $\mathrm{H}-8 \rightarrow \mathrm{C}-(6,7,10), \mathrm{H}-10 \rightarrow \mathrm{C}-(6,8,9 \mathrm{w}, 12), \mathrm{H}-11 \rightarrow \mathrm{C}-(9$, $13,17), \mathrm{H}-13 \rightarrow \mathrm{C}-(11,12,14,15,17), \mathrm{H}-14 \rightarrow \mathrm{C}-(12,16), \mathrm{H}-15$ $\rightarrow$ C- $(13,14,16,17,18 w), \mathrm{H}-18 \rightarrow$ C- $(15,17,20)$, H-19 $\rightarrow$
C-(16, 20, 21), H-22, $23 \rightarrow$ C- $(20,21,22,23)$, H-26 $\rightarrow$ C-(24, $28), \mathrm{H}-27 \rightarrow \mathrm{C}-(25,29,30), \mathrm{H}-29 \rightarrow \mathrm{C}-(21,25,27,30)$, $\mathrm{H}-31 \rightarrow \mathrm{C}-(1,7,32,33), \mathrm{H}-32 \rightarrow \mathrm{C}-(31,33,34), \mathrm{H}-37,45 \rightarrow$ $\mathrm{C}-(36,44,38,46), \mathrm{H}-38,46 \rightarrow \mathrm{C}-(36,44,37,45), \mathrm{H}-39 \rightarrow$ $\mathrm{C}-(20,25,40,41)$. DQF-COSY correlations: $\mathrm{H}-2 \rightarrow \mathrm{H}-(3)$, $\mathrm{H}-3 \rightarrow \mathrm{H}-(2,4), \mathrm{H}-4 \rightarrow \mathrm{H}-(3,5), \mathrm{H}-5 \rightarrow \mathrm{H}-(4), \mathrm{H}-7 \rightarrow$ $\mathrm{H}-(8), \mathrm{H}-8 \rightarrow \mathrm{H}-(7), \mathrm{H}-10 \rightarrow \mathrm{H}-(11), \mathrm{H}-11 \rightarrow \mathrm{H}-(10), \mathrm{H}-13$ $\rightarrow \mathrm{H}-(14), \mathrm{H}-14 \rightarrow \mathrm{H}-(13,15), \mathrm{H}-15 \rightarrow \mathrm{H}-(14), \mathrm{H}-18 \rightarrow$ $\mathrm{H}-(19), \mathrm{H}-19 \rightarrow \mathrm{H}-(18), \mathrm{H}-26 \rightarrow \mathrm{H}-(27), \mathrm{H}-27 \rightarrow \mathrm{H}-(26)$, $\mathrm{H}-31 \rightarrow \mathrm{H}-(32), \mathrm{H}-32 \rightarrow \mathrm{H}-(31,33), \mathrm{H}-33 \rightarrow \mathrm{H}-(32), \mathrm{H}-37$, $45 \rightarrow \mathrm{H}-(38,46), \mathrm{H}-38,46 \rightarrow \mathrm{H}-(37,45), \mathrm{H}-39 \rightarrow \mathrm{H}-(40)$, $\mathrm{H}-40 \rightarrow \mathrm{H}-(39,41), \mathrm{H}-41 \rightarrow \mathrm{H}-(40)$. HR-MS (ESI-QTOF): calculated for $\mathrm{C}_{46} \mathrm{H}_{60} \mathrm{Cl}_{2} \mathrm{~N}_{2} \mathrm{O}_{6}\left[\mathrm{M}-\mathrm{ClO}_{4}\right]^{+}$: 707.4338. Found: 707.4336. Elemental analysis: calculated for $\mathrm{C}_{46} \mathrm{H}_{60} \mathrm{Cl}_{2} \mathrm{~N}_{2} \mathrm{O}_{6}$ : C, 68.39\%; H, 7.49\%; N, 3.47\%; O, 11.88\%; Cl, 8.78\%. Found: C, 68.18\%; H, 7.71\%; N , 3.42\%; O, 12.17\%; Cl, 8.67\%. IR (ATR, $\left.\mathrm{cm}^{-1}\right): \lambda_{\max } 2925,2853,1709,1688,1568,1161,1076,771$.

2.2.6. 2-(2-(3-(2-(5-Carboxy-3,3-dimethyl-1-octylindolin2-ylidene)ethylidene)-2-chlorocyclohex-1-en-1-yl)vinyl)-3ethylbenzo[d]thiazol-3-ium Iodide (Hepta6). Half dye S15 (1.87 mmol, $1.00 \mathrm{~g})$, 5-carboxy-2,3,3-trimethyl-1-octyl-3Hindol-1-ium iodide $\mathbf{S 8}$ (1.87 $\mathrm{mmol}, 0.83 \mathrm{~g})$, and sodium acetate $(4.1 \mathrm{mmol}, 0.33 \mathrm{~g})$ were suspended in ethanol $(50 \mathrm{~mL})$ and heated to reflux for $18 \mathrm{~h}$. After the reaction, the suspension was cooled to room temperature. The precipitated starting materials were removed by filtration. The filtrate was reduced to half under reduced pressure and stored at $-20^{\circ} \mathrm{C}$ for $14 \mathrm{~h}$. Subsequently, the viscose suspension was dropped into hexane $(50 \mathrm{~mL})$ and stored at $4^{\circ} \mathrm{C}$ for $15 \mathrm{~h}$ until fine green shiny crystals precipitated. The crystals were isolated by filtration and recrystallized from ethanol $(30 \mathrm{~mL})$ to yield the dye Hepta6 (0.31 g, 22.5\%). d.p. $255^{\circ}$ C. ${ }^{1} \mathrm{H}$ NMR (DMSO- $\mathrm{d}_{6}$, $400.1 \mathrm{MHz}$ ): $\delta 12.65$ (s, $1 \mathrm{H}, \mathrm{OH}), 8.28(\mathrm{dd}, J=8.1,0.8,1 \mathrm{H}$, H-5), 8.21 (d, $J=14.7,1 \mathrm{H}, \mathrm{H}-9), 8.15$ (d, $J=8.5,1 \mathrm{H}, \mathrm{H}-2), 7.92$ (d, $J=1.6,1 \mathrm{H}, \mathrm{H}-27), 7.87$ (dd, $J=8.3,1.6,1 \mathrm{H}, \mathrm{H}-25), 7.81$ (d, $J=13.2,1 \mathrm{H}, \mathrm{H}-16), 7.79$ (m, 1H, H-3), 7.68 (m, 1H, H-4), 7.14 (d, $J=14.7,1 \mathrm{H}, \mathrm{H}-8), 7.13$ (d, $J=8.3,1 \mathrm{H}, \mathrm{H}-24), 5.89$ (d, $J=$ 13.2, 1H, H-17), 4.81 (q, J = 7.2, 2H, H-29), 3.92 (m, 2H, H-31), $2.74(\mathrm{t}, J=5.9,2 \mathrm{H}, \mathrm{H}-11), 2.66(\mathrm{t}, J=5.9,2 \mathrm{H}, \mathrm{H}-13), 1.84(\mathrm{~m}$, 2H, H-12), 1.64 (m, 2H, H-32), 1.61 (s, 6H, H-20, 21), 1.40 (t, J $=7.2,3 \mathrm{H}, \mathrm{H}-30), 1.33(\mathrm{~m}, 2 \mathrm{H}, \mathrm{H}-33), 1.28$ (m, 4H, H-34, 35), 1.22 (m, 2H, H-37), 1.22 (m, 2H, H-36), 0.83 (t, $J=7.0,3 \mathrm{H}$, $\mathrm{H}-38) .{ }^{13} \mathrm{C}$ NMR (DMSO-d 6 , 100.6 MHz): $\delta 169.5$ (s, C-7), 167.1 (s, C-28), 163.6 (s, C-18), 147.2 (s, C-23), 144.2 (d, C-9), 143.8 (s, C-15), 141.2 (s, C-1), 139.3 (s, C-22), 134.0 (d, C-16), 130.7 (d, C-25), 129.2 (d, C-3), 127.9 (s, C-10), 127.6 (d, C-4), 127.5 (s, C-6), 125.5 (s, C-14), 124.1 (d, C-5), 123.8 (s, C-26), 123.0 (d, C-27), 115.8 (d, C-2), 109.2 (d, C-8), 108.2 (d, C-24), 96.7 (d, C-17), 46.3 (s, C-19), 43.5 (t, C-29), 42.3 (t, C-31), 31.2 (t, C-36), 28.6 (t, C-34, 35), 27.8 (q, C-20, 21), 26.7 (t, C-11), 26.1 (t, C-33), 26.1 (t, C-32), 25.7 (t, C-13), 22.0 (t, C-37), 20.5 (t, C-12), 14.0 (q, C-38), 13.8 (q, C-30). HMBC correlations: $\mathrm{H}-2 \rightarrow \mathrm{C}-(4,6), \mathrm{H}-3 \rightarrow \mathrm{C}-(1,5), \mathrm{H}-4 \rightarrow \mathrm{C}-(2,6), \mathrm{H}-5 \rightarrow$ $\mathrm{C}-(1,3), \mathrm{H}-8 \rightarrow \mathrm{C}-(7,9 \mathrm{w}, 10), \mathrm{H}-9 \rightarrow \mathrm{C}-(7,11,15), \mathrm{H}-11 \rightarrow$ C-(9w, 10, 13, 15), H-12 $\rightarrow$ C-(10, 14), H-13 $\rightarrow$ C-(11, 14, 15, $16 \mathrm{w}), \mathrm{H}-16 \rightarrow \mathrm{C}-(13,18), \mathrm{H}-17 \rightarrow \mathrm{C}-(14,18 \mathrm{w}, 19), \mathrm{H}-20,21$ $\rightarrow$ C- $(18,19,20,21,22,23), \mathrm{H}-24 \rightarrow \mathrm{C}-(22,26), \mathrm{H}-25 \rightarrow$ 
C-(23, 27, 28), H-27 $\rightarrow$ C- $(19,23,25,28)$, H-29 $\rightarrow$ C-(1, 7, $30), \mathrm{H}-30 \rightarrow \mathrm{C}-(29), \mathrm{H}-31 \rightarrow \mathrm{C}-(18,23,33), \mathrm{H}-32 \rightarrow \mathrm{C}-(33$, $34), \mathrm{H}-33 \rightarrow \mathrm{C}-(32,35), \mathrm{H}-34,35 \rightarrow \mathrm{C}-(36), \mathrm{H}-37 \rightarrow \mathrm{C}-(35$, $36)$, H-38 $\rightarrow$ C- $(36,37)$. DQF-COSY correlations: $\mathrm{H}-2 \rightarrow$ $\mathrm{H}-(3), \mathrm{H}-3 \rightarrow \mathrm{H}-(2,4), \mathrm{H}-4 \rightarrow \mathrm{H}-(3,5), \mathrm{H}-5 \rightarrow \mathrm{H}-(4), \mathrm{H}-8$ $\rightarrow \mathrm{H}-(9), \mathrm{H}-9 \rightarrow \mathrm{H}-(8), \mathrm{H}-11 \rightarrow \mathrm{H}-(12), \mathrm{H}-12 \rightarrow \mathrm{H}-(11$, 13), $\mathrm{H}-13 \rightarrow \mathrm{H}-(12), \mathrm{H}-16 \rightarrow \mathrm{H}-(17), \mathrm{H}-17 \rightarrow \mathrm{H}-(16), \mathrm{H}-24$ $\rightarrow \mathrm{H}-(25), \mathrm{H}-25 \rightarrow \mathrm{H}-(24,27), \mathrm{H}-27 \rightarrow \mathrm{H}-(25), \mathrm{H}-29 \rightarrow$ $\mathrm{H}-(30), \mathrm{H}-30 \rightarrow \mathrm{H}-(29), \mathrm{H}-31 \rightarrow \mathrm{H}-(32), \mathrm{H}-32 \rightarrow \mathrm{H}-(31$, $33), \mathrm{H}-33 \rightarrow \mathrm{H}-(32), \mathrm{H}-37 \rightarrow \mathrm{H}-(38), \mathrm{H}-38 \rightarrow \mathrm{H}-(37)$. HR-MS (ESI-QTOF): calculated for $\mathrm{C}_{38} \mathrm{H}_{46} \mathrm{ClN}_{2} \mathrm{O}_{2} \mathrm{~S}[\mathrm{M}-\mathrm{I}]^{+}$: 629.2963. Found: 629.2957. Elemental analysis: calculated for $\mathrm{C}_{38} \mathrm{H}_{46} \mathrm{ClIN}_{2} \mathrm{O}_{2} \mathrm{~S}$ : C, 60.28\%; H, 6.12\%; N, 3.70\%; O, 4.23\%; S, 4.23\%; Cl, 4.68\%; I, 16.76\%. Found: C, 60.02\%; H, 6.16\%; N, 3.72\%; O, 4.48\%; S, 4.16\%; Cl, 4.49\%; I, 16.69\%. IR (ATR, $\left.\mathrm{cm}^{-1}\right): \lambda_{\max } 2923,2852,1705,1604,1548,1359,1096,1049$, 927,754 .

2.2.7. 5-Carboxy-2-(2-(2-chloro-3-(2-(3-ethyl-1,1-dimethyl-1,3dihydro-2H-benzo[e] indol-2-ylidene)ethylidene)cyclohex-1en-1-yl)vinyl)-3,3-dimethyl-1-octyl-3H-indol-1-ium (Hepta7). Half dye S11 (2.55 mmol, $1.52 \mathrm{~g}$ ) and anchor group S8 $(2.9 \mathrm{mmol}, 1.29 \mathrm{~g})$ were dissolved in ethanol $(50 \mathrm{~mL})$ and heated to reflux under argon for $96 \mathrm{~h}$. Then, the mixture was slowly cooled down to $-15^{\circ} \mathrm{C}$ and stored at this temperature overnight. Since no product crystallized the solution was warmed up to room temperature and the desired crude product was isolated by slow crystallization from ethanol/diethyl ether mixture. Further purification by recrystallization from ethanol yielded pure dye Hepta7 $(1.3 \mathrm{~g}, 65 \%) .{ }^{1} \mathrm{H}$ NMR (DMSO-d $\left.6,400.1 \mathrm{MHz}\right): \delta 12.78$ (s (br), $1 \mathrm{H}, \mathrm{OH}), 8.52$ (d, $J=15.0,1 \mathrm{H}, \mathrm{H}-16), 8.38$ (d, $J=8.3$, $1 \mathrm{H}, \mathrm{H}-3), 8.20$ (d, $J=9,1 \mathrm{H}, \mathrm{H}-7), 8.14$ (d, $J=8.4,1 \mathrm{H}, \mathrm{H}-6)$, 8.04 (d, $J=13.5,1 \mathrm{H}, \mathrm{H}-23), 8.01$ (d, $J=1.4,1 \mathrm{H}, \mathrm{H}-34), 7.94$ (d, $J=9,1 \mathrm{H}, \mathrm{H}-8), 7.93$ (dd, $J=8.4,1.4,1 \mathrm{H}, \mathrm{H}-32), 7.72$ (m, 1H, H-4), 7.61 (m, 1H, H-5), 7.28 (d, J = 8.4, 1H, H-31), 6.70 (d, $J=15,1 \mathrm{H}, \mathrm{H}-15), 6.08$ (d, $J=13.5,1 \mathrm{H}, \mathrm{H}-24), 4.56$ (q, $=7.2,2 \mathrm{H}, \mathrm{H}-36), 4.04$ (m, 2H, H-38), 2.77 (m, 2H, H-18), 2.71 (m, 2H, H-20), 1.95 (s, 6H, H-13, 14), 1.87 (m, 2H, H-19), 1.68 (m, 2H, H-39), 1.65 (s, 6H, H-27, 28), 1.42 (t, $J=7.2,3 \mathrm{H}$, H-37), 1.35 (m, 2H, H-40), 1.28 (m, 4H, H-41, 42), 1.24 (m, 2H, H-44), 1.22 (m, 2H, H-43), 0.83 (t, $J=7.1,3 \mathrm{H}, \mathrm{H}-45)$. ${ }^{13} \mathrm{C}$ NMR (DMSO-d $\left.\mathrm{d}_{6}, 100.6 \mathrm{MHz}\right): \delta 177.2(\mathrm{~s}, \mathrm{C}-12), 167.1(\mathrm{~s}$, C-35), 167.1 (s, C-25), 147.1 (s, C-22), 146.5 (s, C-30), 145.6 (d, C-16), 139.9 (s, C-29), 138.7 (s, C-9), 138.0 (d, C-23), 136.1 (s, C-10), 132.2 (s, C-1), 130.8 (d, C-7), 130.6 (d, C-32), 129.8 (d, C-6), 128.1 (d, C-4), 128.0 (s, C-17), 127.0 (s, C-2), 126.3 (s, C-21), 126.1 (d, C-5), 124.9 (s, C-33), 123.1 (d, C-34), 122.7 (d, C-3), 112.2 (d, C-8), 109.3 (d, C-31), 105.6 (d, C-15), 98.6 (d, C-24), 52.0 (s, C-11), 47.2 (s, C-26), 42.8 (t, C-38), 40.6 (t, C-36), 31.1 (t, C-43), 28.5 (t, C-41, 42), 27.6 (q, C-27, 28), 26.6 (q, C-13, 14), 26.3 (t, C-39), 26.0 (t, C-40), 25.9 (t, C-18), 25.7 (t, C-20), 22.0 (t, C-44), 20.3 (t, C-19), 13.9 (q, C-45), 13.0 (q, C-37). HMBC correlations: H-3 $\rightarrow$ C- $(1,5,10), \mathrm{H}-4$ $\rightarrow \mathrm{C}-(2,6), \mathrm{H}-5 \rightarrow \mathrm{C}-(1,3), \mathrm{H}-6 \rightarrow \mathrm{C}-(2,4,7), \mathrm{H}-7 \rightarrow$ C-(1w, 2, 6, 9), H-8 $\rightarrow$ C-(1, 10), H-13, $14 \rightarrow$ C-(10, 11, 12, 13, $14), \mathrm{H}-15 \rightarrow \mathrm{C}-(11,17), \mathrm{H}-16 \rightarrow \mathrm{C}-(12,18 \mathrm{w}, 22), \mathrm{H}-23 \rightarrow$ C-(20w, 22, 25), H-24 $\rightarrow$ C-(21w, 26), H-27, $28 \rightarrow$ C- $(25,26$,
$27,28,29)$, H-31 $\rightarrow$ C-(29, 33), H-32 $\rightarrow$ C-(30, 32w, 34, 35), $\mathrm{H}-34 \rightarrow \mathrm{C}-(30,32,35), \mathrm{H}-37 \rightarrow \mathrm{C}-(36), \mathrm{H}-45 \rightarrow \mathrm{C}-(43$, 44). DQF-COSY correlations: $\mathrm{H}-3 \rightarrow \mathrm{H}-(4), \mathrm{H}-4 \rightarrow \mathrm{H}-(3$, $5), \mathrm{H}-5 \rightarrow \mathrm{H}-(4,6), \mathrm{H}-6 \rightarrow \mathrm{H}-(5), \mathrm{H}-7 \rightarrow \mathrm{H}-(8), \mathrm{H}-8 \rightarrow$ $\mathrm{H}-(7), \mathrm{H}-15 \rightarrow \mathrm{H}-(16), \mathrm{H}-16 \rightarrow \mathrm{H}-(15), \mathrm{H}-18 \rightarrow \mathrm{H}-(19)$, $\mathrm{H}-19 \rightarrow \mathrm{H}-(18,20), \mathrm{H}-20 \rightarrow \mathrm{H}-(19), \mathrm{H}-23 \rightarrow \mathrm{H}-(24)$, $\mathrm{H}-24 \rightarrow \mathrm{H}-(23), \mathrm{H}-31 \rightarrow \mathrm{H}-(32), \mathrm{H}-32 \rightarrow \mathrm{H}-(31), \mathrm{H}-36 \rightarrow$ $\mathrm{H}-(37), \mathrm{H}-37 \rightarrow \mathrm{H}-(36), \mathrm{H}-38 \rightarrow \mathrm{H}-(39), \mathrm{H}-39 \rightarrow \mathrm{H}-(38$, $40), \mathrm{H}-40 \rightarrow \mathrm{H}-(39), \mathrm{H}-44 \rightarrow \mathrm{H}-(45), \mathrm{H}-45 \rightarrow \mathrm{H}-(44)$. HR-MS (ESI-QTOF): calculated for $\mathrm{C}_{45} \mathrm{H}_{54} \mathrm{ClN}_{2} \mathrm{O}_{2}[\mathrm{M}-\mathrm{I}]^{+}$: 689.3868. Found: 689.3854.

2.3. DSC Preparation. State-of-art cells were prepared with several titania photo anodes $\left(0.36 \mathrm{~cm}^{2}\right)$ printed on FTO glass $(2.2 \mathrm{~mm}$ thick and $7 \mathrm{Ohm} / \mathrm{sq})$. The layering used for high efficiency cells was as follows (see Supplementary Figure S2): around $12 \mu \mathrm{m}$ of $18 \mathrm{~nm} \mathrm{TiO}_{2}$ nanoparticles (Solaronix TiNanoxide T/SP) and $3 \mu \mathrm{m}$ of over $100 \mathrm{~nm} \mathrm{TiO}{ }_{2}$ nanoparticles (Solaronix Ti-Nanoxide R/SP). For testing purpose sometimes $4 \mu \mathrm{m}$ thick layers of $18 \mathrm{~nm} \mathrm{TiO}_{2}$ nanoparticles were used without addition of the scattering layer.

$\mathrm{TiCl}_{4}$ pretreatment and posttreatment were performed to optimize titania's adhesion, porosity, and specific area. The counter electrode was prepared by paint brushing three layers of Solaronix Platisol T, leading to the formation of a platinized catalytic layer after firing at $400^{\circ} \mathrm{C}$. The photoanodes were then sensitized using the various dyes in different solvents with or without chenodeoxycholic acid as coadsorbent to limit dye aggregation. The working and counter electrodes were joined together using a $60 \mu \mathrm{m}$ spacer (or $25 \mu \mathrm{m}$ for $4 \mu \mathrm{m}$ thick electrodes). The cells were then backfilled with an electrolyte based on acetonitrile containing $0.5 \mathrm{M}$ of dimethyl propyl imidazolium iodide, $0.5 \mathrm{M}$ of 4 - $t$-butylpyridine, $0.1 \mathrm{M}$ of lithium iodide, and $50 \mathrm{mM}$ of iodine. For accurate measurement the cells were masked. The total active area was $0.36 \mathrm{~cm}^{2}$.

2.4. Large-Area DSC Preparation with Hepta4. Thickness of the $\mathrm{TiO}_{2}$ layer was $14 \mu \mathrm{m}$ with an additional scatter layer of $4 \mu \mathrm{m}$. For immersion, the optimal concentration was $0.5 \mathrm{mM}$ for Hepta4 and $10 \mathrm{mM}$ for CDCA in methanol. The electrolyte was AN50 from Solaronix with a concentration of $50 \mathrm{mmol}$ of $\mathrm{I}^{-} / \mathrm{I}_{3}{ }^{-}$in acetonitrile.

2.5. Characterization of the DSCs. The DSCs were characterized using a SolarSim 150 (Solaronix) previously calibrated. The measurements were carried out under one sun $\left(1000 \mathrm{~W} / \mathrm{m}^{2}, \mathrm{AM}-1.5 \mathrm{G}\right)$ on masked cells (active area $\left.0.36 \mathrm{~cm}^{2}\right)$.

\section{Results and Discussion}

3.1. Synthetic Concept. Commonly, symmetrical heptamethine dyes have been synthesized via Knoevenagel condensation reaction between heterocyclic bases with an activated methyl group and an unsaturated bisaldehyde or the dianil derivative in the presence of catalyst, for example, anhydrous sodium acetate or trimethylamine. Commonly used solvents 


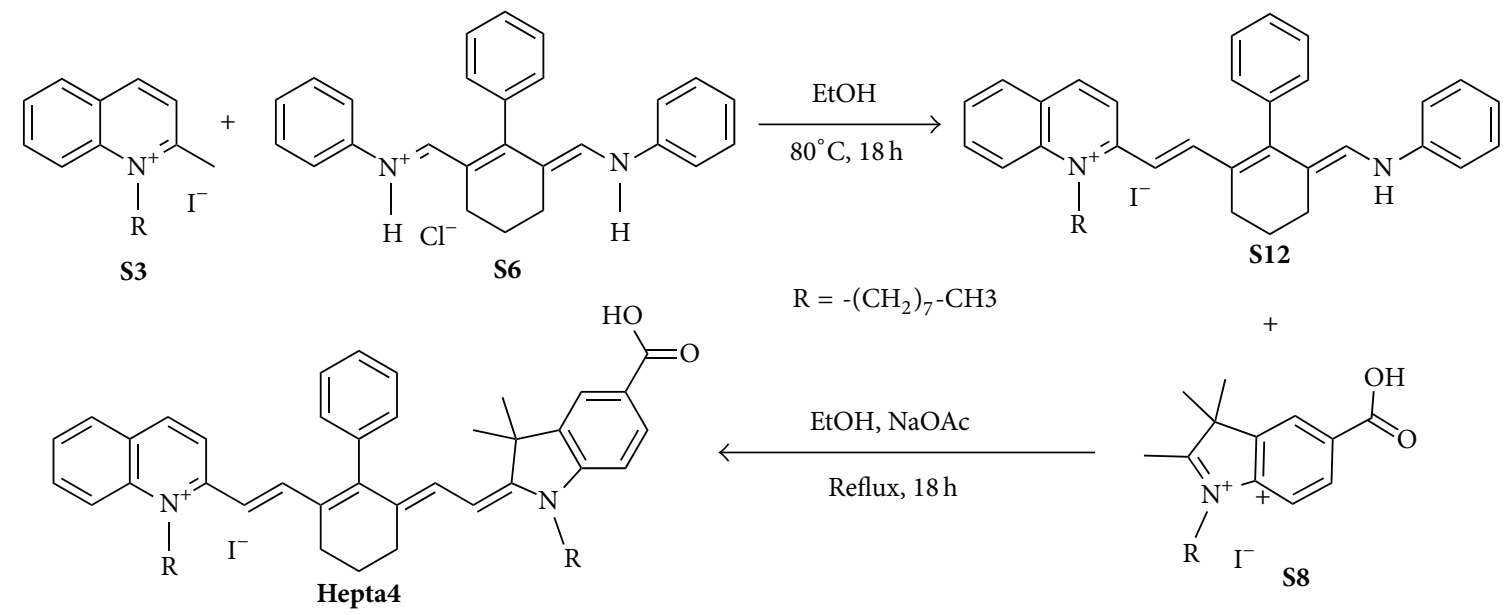

SCHEme 1: Synthetic route of Hepta4.

for the reactions are alcohols, acetic acids, acetic anhydride, or mixtures of them. By adapting this reaction condition and stepwise condensation reaction via a half dye intermediate, unsymmetrical heptamethine dyes are achievable. Remarkably, despite equimolar amounts of the starting materials, the use of a catalyst often causes symmetrical side products in the first step which entails elaborate purification by column chromatography. Here, the first Knoevenagel condensation reaction step was carried out at equimolar conditions in anhydrous ethanol under inert gas without catalyst at reflux temperature. The activity of the alkylated heterocycles S1S5 (see Supplementary Material) is sufficient enough to selectively react with the corresponding dianils S6 and S7 to its half dyes S11-S16 while the second condensation reaction step to the symmetrical full dye is prevented. The half dyes precipitate in the reaction mixture directly or after chilling and can be further purified by recrystallization. The anchor moiety (the second heterocycle $\mathbf{S 8}$ or $\mathbf{S 1 0}$ ) is condensed to the corresponding half dye via a second Knoevenagel reaction step under catalyzed condition (anhydrous sodium acetate) in anhydrous ethanol. The resulting unsymmetrical heptamethine dyes Hepta1-Hepta7 also precipitate in the reaction solution and can be further purified via recrystallization. As an example, a detailed reaction scheme is given for the synthesis of Hepta4 (Scheme 1).

3.2. Optical Properties. Optical data and UV-vis spectra in ethanol of the unsymmetrical heptamethine dyes HeptalHepta7 and, for comparison, symmetrical heptamethine dyes symHeptal-symHepta3 are provided in Table 1 and Supplementary Material. In general, heptamethine dyes are characterized by their specific spectral properties exhibiting highly intense and narrow absorption bands. Seven methine units and large aromatic terminals lead to bathochromically shifted absorption in the NIR domain. The extraordinary optical properties furthermore require a uniform $\mathrm{C}-\mathrm{C}$ bond length (no bond-length alternation), the maximum alternation of the positive and negative partial charge of the carbon atoms in the polymethine chain, and a high symmetry of the dye chromophore [20]. The symmetrical dyes symHeptal-symHepta3 with identical structural and chemical termination achieve the cyanine limit resulting in maximum bathochromy of $\lambda_{\max }$ and highest values of $\varepsilon$. In the case of Hepta7, the electronic structure of the polymethine chain remains unaffected by the additional carboxylic acid function. Thus, the dye exhibits the same optical properties as its corresponding symmetrical dye. The unsymmetrical combination of heterocycles with different basicity in Heptal, Hepta2, Hepta3, Hepta4, and Hepta6 causes a symmetry break resulting in a slight hypsochromic shift of the $\lambda_{\max }$ value, a noticeable decrease of the extinction coefficient $\varepsilon$ at $\lambda_{\text {max }}$, and additional weak absorption at around $400 \mathrm{~nm}$. In principal, Hepta5 has to be classified as nonamethine dye because of additional methine units between the nitrogen atoms inducing a distinct hypsochromic shift of $\lambda_{\max }$ of almost $100 \mathrm{~nm}$ and the lowest $\varepsilon$ value at $\lambda_{\max }$ of all unsymmetrical dyes. For almost all dyes, the value of the oscillator strength $f$ is greater than unity because more than one electron is involved in the optical transition and the electronic system is somehow degenerated.

3.3. Electrochemical Properties. Cyclic voltammetry measurements were performed for all unsymmetrical dyes in DMF solution. The results are presented in Table 2 and the voltammograms are shown in the Supplementary Material. For most dyes, broad and irreversible oxidation and reduction waves were observed within the potential range of DMF. According to the literature [21], all unsymmetrical dyes undergo an irreversible oxidation and their radical dications couple to form stable dimers. Therefore, the first reduction and oxidation potential $\left(E_{\mathrm{red}}, E_{\mathrm{ox}}\right)$ were roughly estimated from the corresponding anodic or cathodic peak potentials. Remarkably, symmetrical dyes exhibit reversible oxidation and reduction processes (e.g., see voltammogram of symHepta2 in Supplementary Material). With regard to application in DSC, efficient electron injection from the reduction level of the dye into the conduction band of the semiconductor is mandatory. But, the reduction potential of 
TABLE 1: Optical properties of the unsymmetrical and symmetrical heptamethine dyes in ethanol.

\begin{tabular}{lccccc}
\hline Dye $^{\mathrm{a}}$ & $\lambda_{\max }(\mathrm{nm})^{\mathrm{b}}$ & $\varepsilon\left(\lambda_{\max }\right)\left(\mathrm{Lcm}^{-1} \mathrm{~mol}^{-1}\right)^{\mathrm{c}}$ & $f^{\mathrm{d}}$ & $\lambda_{\text {onset }}^{\text {abs }}(\mathrm{nm})^{\mathrm{e}}$ & 882 \\
\hline Hepta1 & 790 & 133000 & 1.8 & 888 & $E_{g}^{\text {opt }}(\mathrm{eV})^{\mathrm{f}}$ \\
Hepta2 & 795 & 100000 & 1.5 & 866 & 1.41 \\
Hepta3 & 754 & 94600 & 1.7 & 852 & 1.40 \\
Hepta4 & 758 & 82500 & 1.4 & 896 \\
Hepta5 & 702 & 51700 & 0.9 & 830 & 1.46 \\
Hepta6 & 788 & 108000 & 1.5 & 847 & 1.38 \\
Hepta7 & 810 & 240000 & 1.3 & 894 \\
symHepta1 & 850 & 262000 & 1.9 & 830 \\
symHepta2 & 797 & 357000 & 2.0 & 1.49 \\
symHepta3 & 799 & 227000 & 1.3 & 1.39 \\
\hline
\end{tabular}

${ }^{a}$ Chemical structures of the symmetrical heptamethine dyes are given in the Supplementary Material. ${ }^{b}$ Absorption maximum, UV-vis spectra of all dyes are represented in the Supplementary Material. ${ }^{c}$ Molar absorption coefficient determined from the absorbance for a molar concentration $\left(c \approx 2 \times 10^{-6} \mathrm{molL}^{-1}\right)$ of the dyes with a path length of $1 \mathrm{~cm}$ at $\lambda_{\max } .{ }^{\mathrm{d}}$ Oscillator strength, $f=4.31 \times 10^{-9} \int \varepsilon(\widetilde{v}) d \widetilde{v}$. ${ }^{\mathrm{e}}$ Onset absorption edge at higher wavelengths. ${ }^{\mathrm{f}}$ Optical band gap, $E_{g}^{\text {opt }}=\mathrm{hc} / \lambda_{\text {onset }}^{\text {abs }}$.

TABLE 2: Electrochemical properties of the unsymmetrical heptamethine dyes in dilute DMF solution ${ }^{\mathrm{a}}$.

\begin{tabular}{lcccc}
\hline Dye & $E_{\text {ox }}(\mathrm{V} \text { versus NHE })^{\mathrm{b}}$ & $E_{\text {red }}(\mathrm{V} \text { versus NHE })^{\mathrm{c}}$ & $E_{g}^{\mathrm{el}}(\mathrm{V})^{\mathrm{d}}$ & 1.16 \\
Hepta1 & 0.75 & -0.42 & 1.21 & -0.65 \\
Hepta2 & 0.75 & -0.47 & 0.91 & -0.63 \\
Hepta3 & 0.56 & -0.35 & 1.23 & -0.85 \\
Hepta4 & 0.77 & -0.46 & 1.12 & -0.65 \\
Hepta5 & 0.75 & -0.37 & 0.88 & -0.70 \\
Hepta6 & 0.59 & -0.29 & 1.11 & -0.88 \\
Hepta7 & 0.90 & -0.21 & -0.55 \\
\hline
\end{tabular}

${ }^{\mathrm{a}}$ All potentials are referenced to NHE by adopting a potential of $+0.72 \mathrm{~V}$ versus NHE for $\mathrm{Fc} / \mathrm{Fc}^{+}$in DMF [19]. ${ }^{\mathrm{b}} E_{\mathrm{ox}}=\left|E_{\mathrm{pa}, \mathrm{ox}}-E_{\mathrm{pc}, \mathrm{ox}}\right| / 2$ with the cathodic and anodic peak potentials $E_{\mathrm{pa}}$ and $E_{\mathrm{pc}}$ from the oxidation wave. ${ }^{\mathrm{c}} E_{\mathrm{red}}=\left|E_{\mathrm{pa}, \mathrm{red}}-E_{\mathrm{pc} \text {,red }}\right| / 2$ with the cathodic and anodic peak potentials $E_{\mathrm{pa}}$ and $E_{\mathrm{pc}}$ from the reduction wave. ${ }^{\mathrm{d}} E_{g}^{\mathrm{el}}=E_{\mathrm{ox}}-E_{\text {red }}$.

all dyes is more positive than the conduction band edge of the semiconductor $\mathrm{TiO}_{2}(-0.5 \mathrm{~V})$. Charge injection may still be possible from the excited state of the dye, since the latter generally provides more driving force for the electron transfer step (Table 2, $E_{\text {ox }}-E_{g}^{\text {opt }}$ ). Efficient regeneration of the dye after excitation is guaranteed by the sufficiently large difference between the potential of the redox couple $\mathrm{I}^{-} / \mathrm{I}_{3}{ }^{-}(0.4 \mathrm{~V})$ and the oxidation potential of the dyes.

3.4. Molecular Modelling. The molecular structure and electron density distribution provide detailed information about possible vectorial electron transfer from the light-harvesting dye toward the semiconductor surface. Therefore, density functional calculations were performed in silico at B3LYP/6$31 \mathrm{G}^{*}$ level with NWChem [22]. The frontier orbitals of the fully optimized dyes are shown in the Supplementary Material. Typically, a donor- $\pi$ bridge-acceptor $(D-\pi-A)$ dye structure favors the intramolecular charge transfer after excitation from subunit $\mathrm{D}$ through the $\pi$-bridge to $\mathrm{A}$ and the anchor functionality into the semiconductor. Remarkably, none of the unsymmetrical heptamethine dyes correspond to this $\mathrm{D}-\pi$-A structure, and their electron density distribution of the LUMO level is mainly localized within the dye core. Thus, it is assumed that the electron injection after excitation occurs preferentially over the $\pi$ electron system of the dye core instead of through the carboxylic acid anchor.

3.5. NIR Dye-Sensitized Solar Cells. For a proof of principle, all unsymmetrical heptamethine dyes were validated in standard DSC configurations with $\mathrm{TiO}_{2}$ as semiconductor and $\mathrm{I}^{-} / \mathrm{I}_{3}{ }^{-}$as redox couple. In order to prevent the dye from aggregation on the semiconductor surface, chenodeoxycholic acid (CDCA) was used as coadsorbent. Due to previous adsorption experiments with different solvent combinations, CDCA concentrations, immersing temperatures, and times, optimal parameters for the dye immobilization were evaluated. Surprisingly, only DSCs with Hepta4 as sensitizer exhibit a noteworthy overall conversion efficiency $(\eta)$ of $1.22 \%$ with a short circuit photocurrent density $\left(J_{\mathrm{SC}}\right)$ of $3.22 \mathrm{~mA} \mathrm{~cm}^{-2}$, an open circuit voltage $\left(V_{\mathrm{OC}}\right)$ of $0.48 \mathrm{~V}$, and a fill factor (FF) of 0.78 (active area $0.36 \mathrm{~cm}^{2}$ ). The $I-V$ curve of this DSC is shown in Figure 2. Similar efficiency values for DSC with symmetrical heptamethines are reported in the literature [6-11]. 


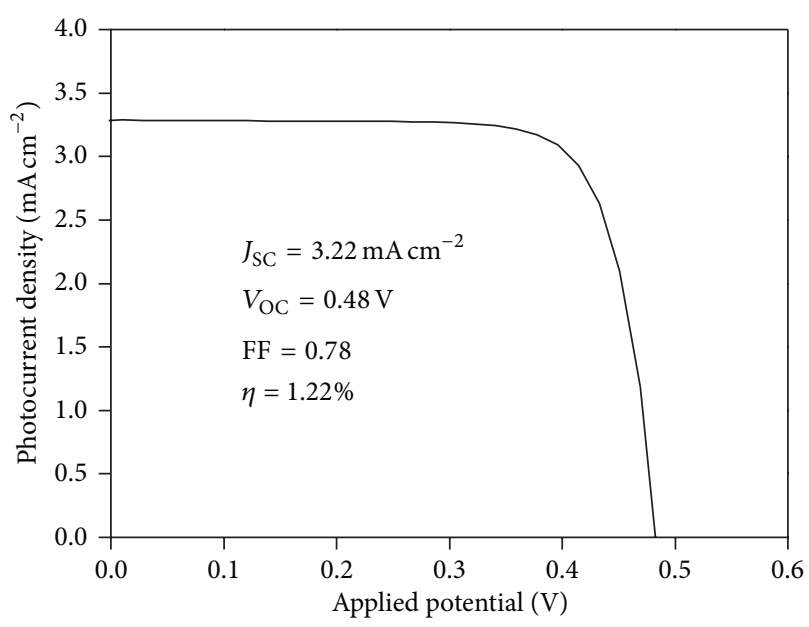

Figure 2: $I-V$ curve of a DSC with Hepta4 as sensitizer.

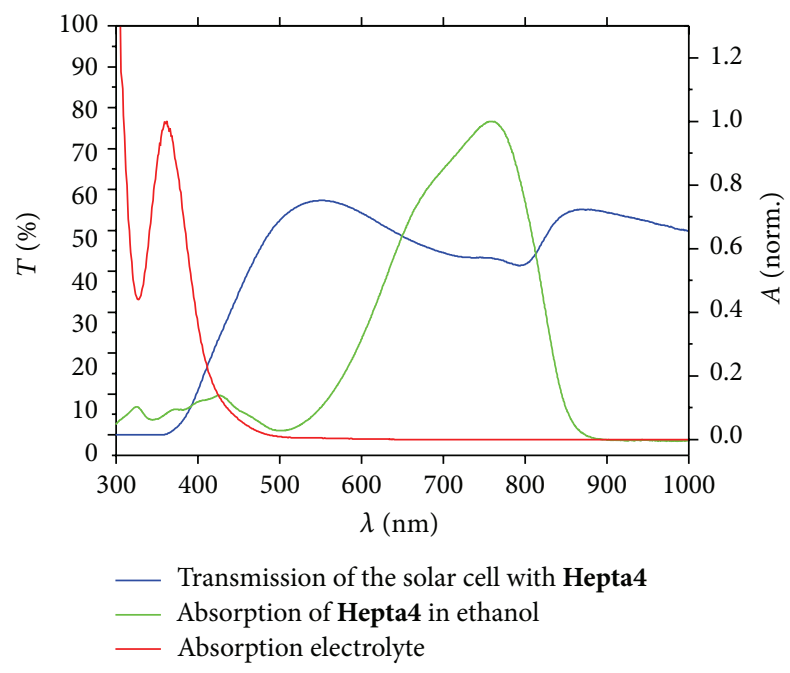

FIgURE 3: Transmission of large-area DSC with NIR dye.

All other unsymmetrical dyes fail under applied conditions in the DSC exhibiting very low or negligible $J_{S C}$ values. Actually, it is not obvious that only Hepta4 is suitable for an application in DSC.

Although data from electrochemical measurements and calculated electron density distributions of the LUMO level strongly indicate unfavourable injection conditions, electrons are nevertheless injected efficiently upon excitation from Hepta4 into the semiconductor conduction band. This underlines the above mentioned interaction of the $\pi$ electrons in the LUMO level with the semiconductor surface as probable electron transfer route.

A nonoptimized large-area cell $\left(90 \mathrm{~cm}^{2}, \eta<1 \%\right)$ with Hepta4 as sensitizer was prepared in order to prove the NIR absorption concept. Figure 3 presents the UV-vis absorption spectrum of Hepta4 as well as the electrolyte in solution and the transmission spectrum of the complete solar cell. Figure 4 provides a photograph of this DSC. The color of the solar cell is pale green and the cell itself is highly transparent. Because

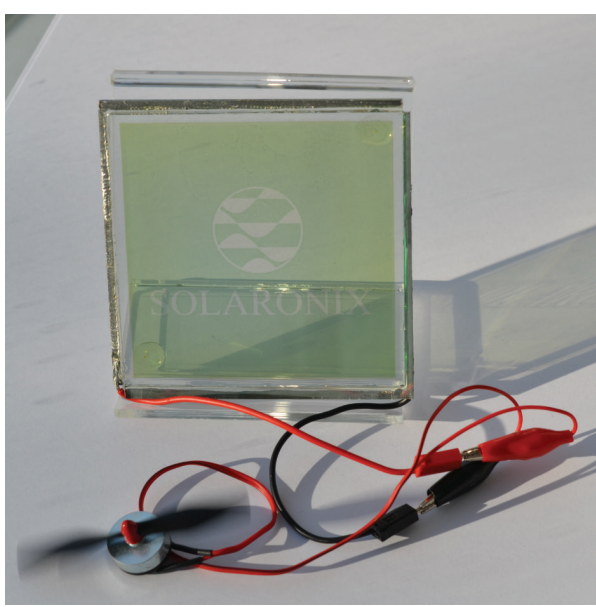

FIGURE 4: Photograph of a working large-area DSC with Hepta4.

of the NIR absorption property of Hepta4, a transmittance of $40-55 \%$ in the visible range of $400-700 \mathrm{~nm}$ is achievable. The reduction of the transmittance occurs because of the absorption band of the electrolyte at 300-500 nm and the extensive tailing of the absorption band of the dye down to $500 \mathrm{~nm}$. Here, an improvement could be achieved by the application of a colorless electrolyte system $(\lambda<350 \mathrm{~nm})$ and a dye with $\lambda_{\max }>900 \mathrm{~nm}$. Nevertheless, the high potential of the unsymmetrical heptamethine dyes for transparent and colorless DSC could be outlined.

\section{Conclusions}

In summary, we have synthesized and characterized seven different unsymmetrical heptamethine dyes equipped with one carboxylic acid anchor group. The use of chemically different heterocycles terminating the polymethine core causes a symmetry break with the strongest influence on the optical properties. Although the redox level of the dyes and the calculated electron density contribution let us suspect limited usability of the dyes, Hepta4 exhibits a notable overall conversion efficiency of $1.22 \%$ in a DSC with $\mathrm{TiO}_{2}$ as semiconductor. The synthesis of dyes with $\lambda_{\max }>900 \mathrm{~nm}$ and different counter ions and the preparation of solar cells with different semiconductors (e.g., $\mathrm{SnO}_{2}$ ) and electrolytes as well as lifetime tests are still ongoing work.

Supplementary Material available: synthetic procedures and characterization of precursors and half dyes, UV-vis spectra, voltammograms, frontier orbitals of the unsymmetrical heptamethines, and a SEM cross section image of a DSC are provided.

\section{Conflict of Interests}

The authors declare that there is no conflict of interests regarding the publication of this paper. 


\section{Acknowledgments}

The authors acknowledge the support of the Commission for Technology and Innovation (CTI), Simone Hochleitner (CV, UV-vis), Beatrice Fischer (DSC, TGA), and Daniele Passerone (molecular modeling) [23].

\section{References}

[1] S. Daehne, U. Resch-Genger, and O. S. Wolfbeis, "Near-infrared dyes for High Technology Applications," in NATO ASI Series, vol. 52 of Series 3, Kluwer Academic, Boston, Mass, USA, 1998.

[2] J. Fabian, H. Nakazumi, and M. Matsuoka, "Near-infrared absorbing dyes," Chemical Reviews, vol. 92, no. 6, pp. 1197-1216, 1992.

[3] C. Qin, W. Y. Wong, and L. Han, "Squaraine dyes for dyesensitized solar cells: recent advances and future challenges," Chemistry, vol. 8, no. 8, pp. 1706-1719, 2013.

[4] J. Park, G. Viscardi, C. Barolo, and N. Barbero, "Near-infrared sensitization in dyesensitized solar cells," Chimia, vol. 67, no. 3, pp. 129-135, 2013.

[5] N. Tyutyulkov, J. Fabian, A. Mehlhorn, F. Dietz, and A. Tadjer, Polymethine Dyes Structure and Properties, St. Kliment Ohridski University Press, Sofia, Bulgaria, 1991.

[6] K. Sayama, K. Hara, Y. Ohga, A. Shinpou, S. Suga, and H. Arakawa, "Significant effects of the distance between the cyanine dye skeleton and the semiconductor surface on the photoelectrochemical properties of dye-sensitized porous semiconductor electrodes," New Journal of Chemistry, vol. 25, no. 2, pp. 200-202, 2001.

[7] M. Matsui, Y. Hashimoto, K. Funabiki, J.-Y. Jin, T. Yoshida, and H. Minoura, "Application of near-infrared absorbing heptamethine cyanine dyes as sensitizers for zinc oxide solar cell," Synthetic Metals, vol. 148, no. 2, pp. 147-153, 2005.

[8] A. Otsuka, K. Funabiki, N. Sugiyama et al., "Design and synthesis of near-infrared-active heptamethine-cyanine dyes to suppress aggregation in a dye-sensitized porous zinc oxide solar cell," Chemistry Letters, vol. 37, no. 2, pp. 176-177, 2008.

[9] T. Ono, T. Yamaguchi, and H. Arakawa, "Study on dyesensitized solar cell using novel infrared dye," Solar Energy Materials and Solar Cells, vol. 93, no. 6-7, pp. 831-835, 2009.

[10] K. Funabiki, H. Mase, A. Hibino et al., "Synthesis of a novel heptamethine-cyanine dye for use in near-infrared active dyesensitized solar cells with porous zinc oxide prepared at low temperature," Energy and Environmental Science, vol. 4, no. 6, pp. 2186-2192, 2011.

[11] E. A. McArthur, J. M. Godbe, D. B. Tice, and E. A. Weiss, "A study of the binding of cyanine dyes to colloidal quantum dots using spectral signatures of dye aggregation," Journal of Physical Chemistry C, vol. 116, no. 10, pp. 6136-6142, 2012.

[12] Y. Shi, R. B. M. Hill, J.-H. Yum et al., "A high-efficiency panchromatic squaraine sensitizer for dye-sensitized solar cells," Angewandte Chemie, vol. 50, no. 29, pp. 6619-6621, 2011.

[13] L. Beverina, R. Ruffo, C. M. Mari et al., "Panchromatic crosssubstituted squaraines for dye-sensitized solar cell applications," ChemSusChem, vol. 2, no. 7, pp. 621-624, 2009.

[14] C. Magistris, S. Martiniani, N. Barbero et al., "Near-infrared absorbing squaraine dye with extended $\pi$ conjugation for dyesensitized solar cells," Renewable Energy, vol. 60, pp. 672-678, 2013.
[15] S. Martiniani, A. Y. Anderson, C. Law, B. C. O’Regan, and C. Barolo, "New insight into the regeneration kinetics of organic dye sensitised solar cells," Chemical Communications, vol. 48, no. 18, pp. 2406-2408, 2012.

[16] J. H. Yum, P. Walter, S. Huber et al., "Efficient far red sensitization of nanocrystalline $\mathrm{TiO}_{2}$ films by an unsymmetrical squaraine dye," Journal of the American Chemical Society, vol. 129, no. 34, pp. 10320-10321, 2007.

[17] T. Geiger, S. Kuster, J.-H. Yum et al., "Molecular design of unsymmetrical squaraine dyes for high efficiency conversion of low energy photons into electrons using $\mathrm{TiO}_{2}$ nanocrystalline films," Advanced Functional Materials, vol. 19, no. 17, pp. 2720 2727, 2009.

[18] S. Kuster, F. Sauvage, M. K. Nazeeruddin, M. Grätzel, F. A. Nüesch, and T. Geiger, "Unsymmetrical squaraine dimer with an extended $\pi$-electron framework: an approach in harvesting near infra-red photons for energy conversion," Dyes and Pigments, vol. 87, no. 1, pp. 30-38, 2010.

[19] W. C. Barrette Jr., H. W. Johnson Jr., and D. T. Sawyer, "Voltammetric evaluation of the effective acidities (pKa') for Broensted acids in aprotic solvents," Analytical Chemistry, vol. 56, no. 11, pp. 1890-1898, 1984.

[20] A. D. Kachkovskii, "The nature of electronic transitions in linear conjugated systems," Russian Chemical Reviews, vol. 66, no. 8, pp. 647-664, 1997.

[21] J. R. Lenhard and A. D. Cameron, "Electrochemistry and electronic spectra of cyanine dye radicals in acetonitrile," Journal of Physical Chemistry, vol. 97, no. 19, pp. 4916-4925, 1993.

[22] M. Valiev, E. J. Bylaska, N. Govind et al., "NWChem: a comprehensive and scalable open-source solution for large scale molecular simulations," Computer Physics Communications, vol. 181, no. 9, pp. 1477-1489, 2010.

[23] 2014, http://www.ipazia.ch. 

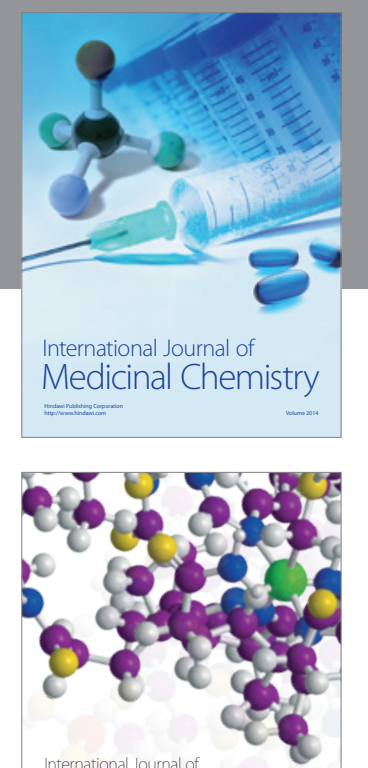

\section{Carbohydrate} Chemistry

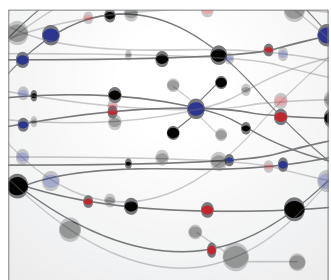

The Scientific World Journal
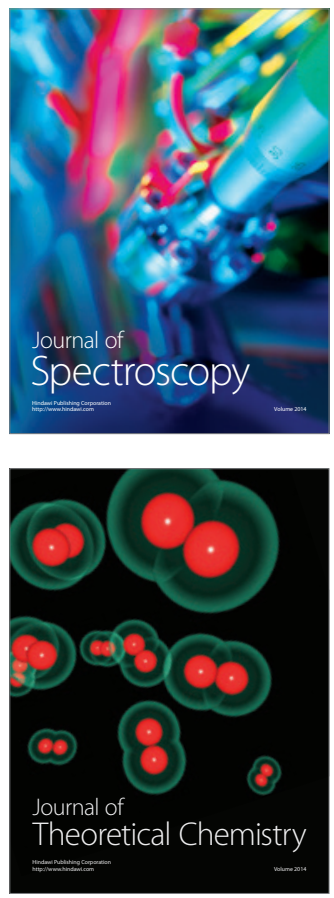
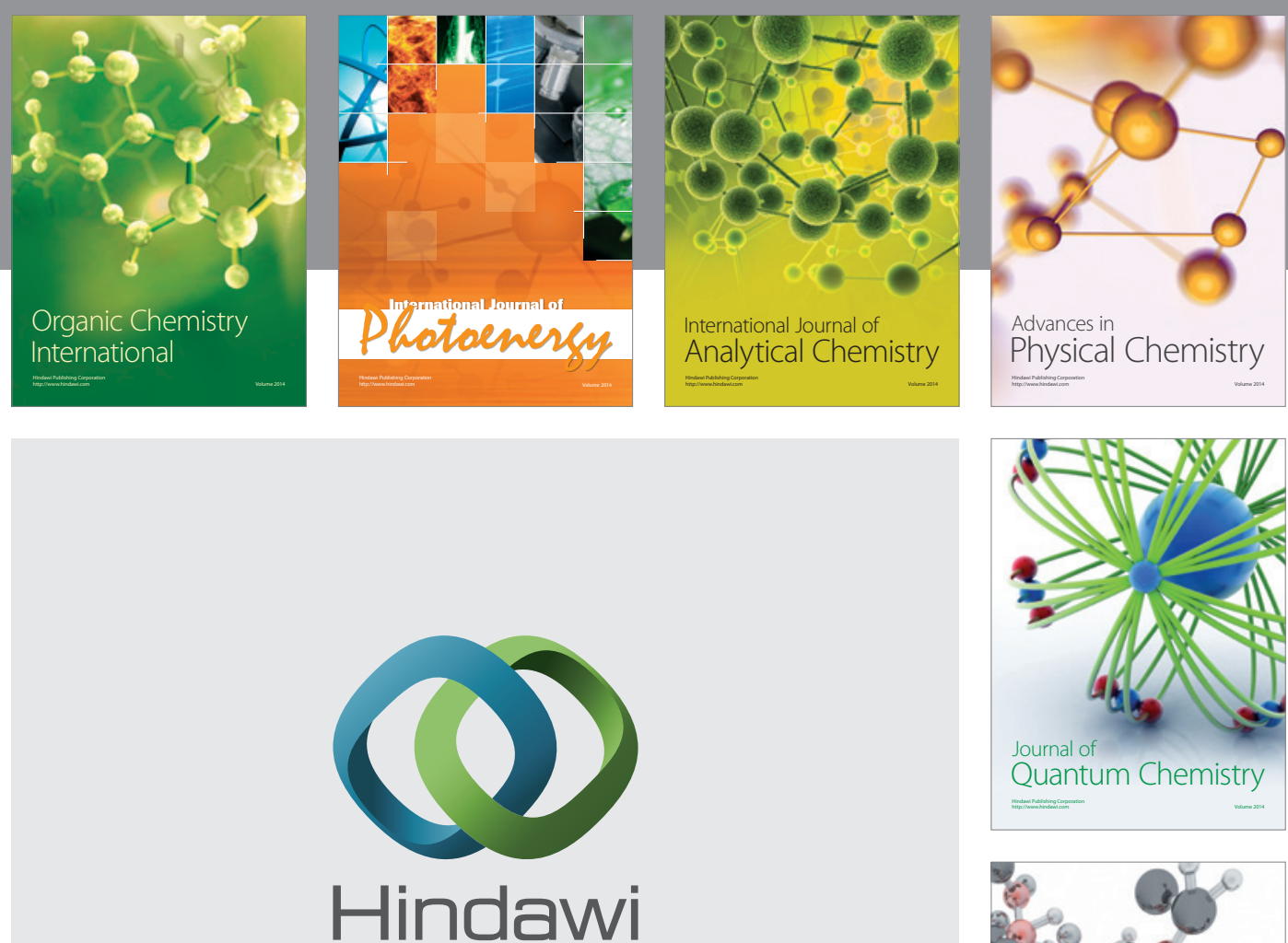

Submit your manuscripts at

http://www.hindawi.com

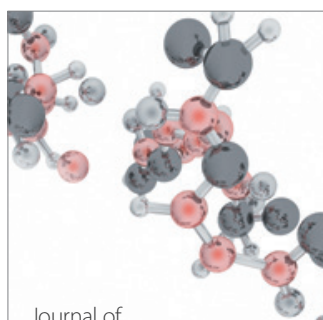

Analytical Methods

in Chemistry

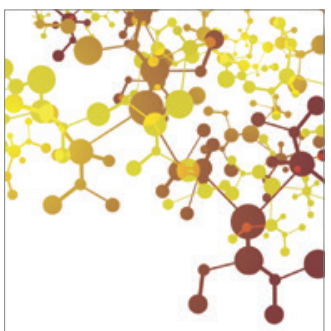

Journal of

Applied Chemistry

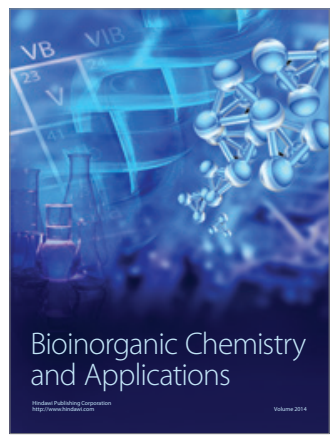

Inorganic Chemistry
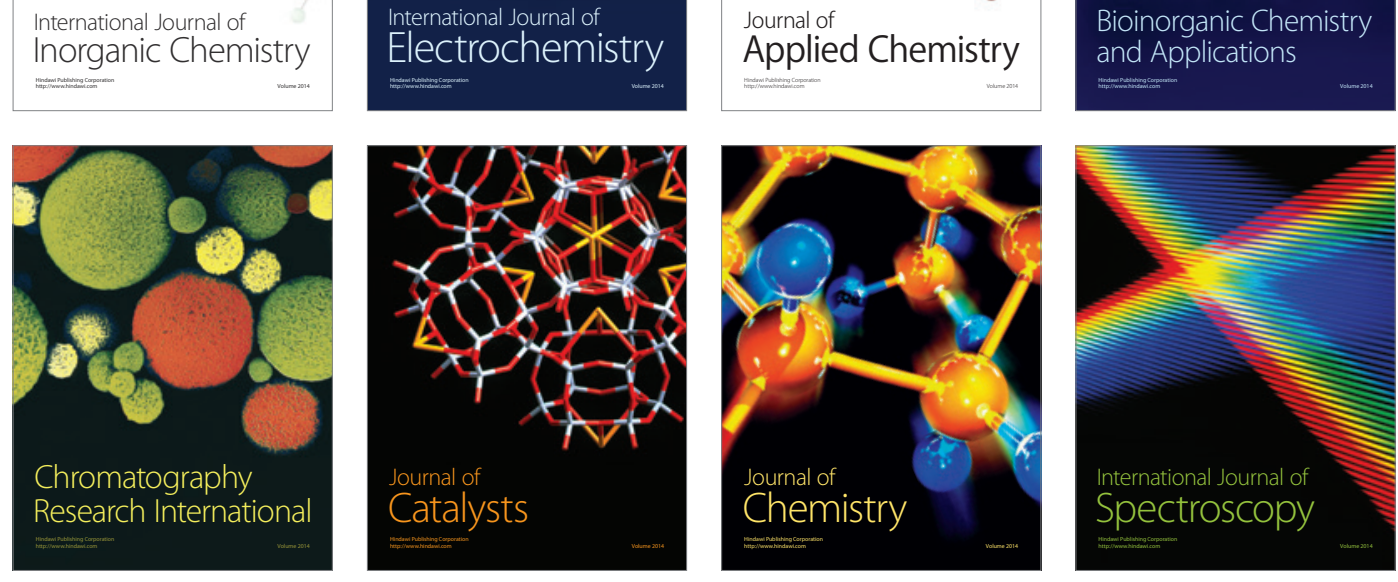